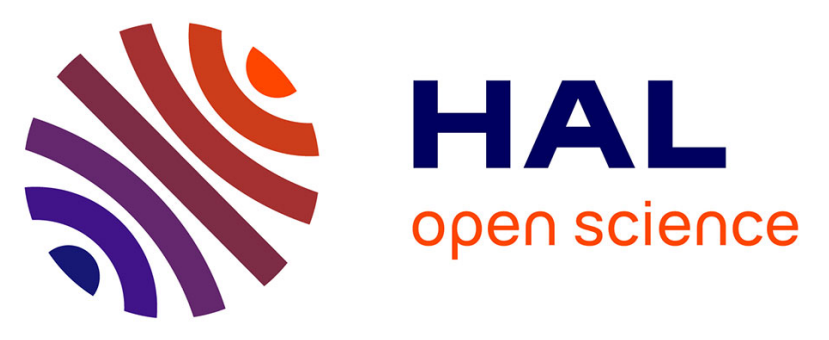

\title{
Palladium-Catalysed Desulfitative Heck Reaction Tolerant to Aryl Carbon-Halogen Bonds for Access to (Poly)halo-Substituted Stilbene or Cinnamate Derivatives
}

Aymen Skhiri, Ridha Ben Salem, Jean-François Soulé, Henri Doucet

\section{To cite this version:}

Aymen Skhiri, Ridha Ben Salem, Jean-François Soulé, Henri Doucet. Palladium-Catalysed Desulfitative Heck Reaction Tolerant to Aryl Carbon-Halogen Bonds for Access to (Poly)halo-Substituted Stilbene or Cinnamate Derivatives. Synthesis: Journal of Synthetic Organic Chemistry, 2016, 48 (18), pp.3097-3106. 10.1055/s-0035-1560449 . hal-01367223

HAL Id: hal-01367223

https://hal-univ-rennes1.archives-ouvertes.fr/hal-01367223

Submitted on 10 Oct 2016

HAL is a multi-disciplinary open access archive for the deposit and dissemination of scientific research documents, whether they are published or not. The documents may come from teaching and research institutions in France or abroad, or from public or private research centers.
L'archive ouverte pluridisciplinaire HAL, est destinée au dépôt et à la diffusion de documents scientifiques de niveau recherche, publiés ou non, émanant des établissements d'enseignement et de recherche français ou étrangers, des laboratoires publics ou privés. 


\section{Pd-catalysed desulfitative Heck reaction tolerant to aryl C-Halo bonds for access to (poly)halo-substituted stilbene or cinnamate derivatives}

\author{
Aymen Skhiri ${ }^{a, b}$ \\ Ridha Ben Salem*b \\ Jean-Francois Soulé ${ }^{a}$ \\ Henri Doucet*a \\ a Institut Sciences Chimiques de Rennes, UMR 6226 CNRS- \\ Université de Rennes "Organométalliques: Matériaux et \\ Catalyse", Campus de Beaulieu, 35042 Rennes, France. Fax: \\ +33-(0)2-23-23-69-39; Tel: +33-(0)2-23-23-63-84. \\ b Laboratoire de Chimie Organique Physique (UR 11ES74) \\ Université de Sfax, Faculté des Sciences de Sfax, Route de la \\ Soukra km 4, 3038 Sfax, Tunisie. \\ ridhabensalem@yahoo.fr; henri.doucet@univ-rennes1.fr
}

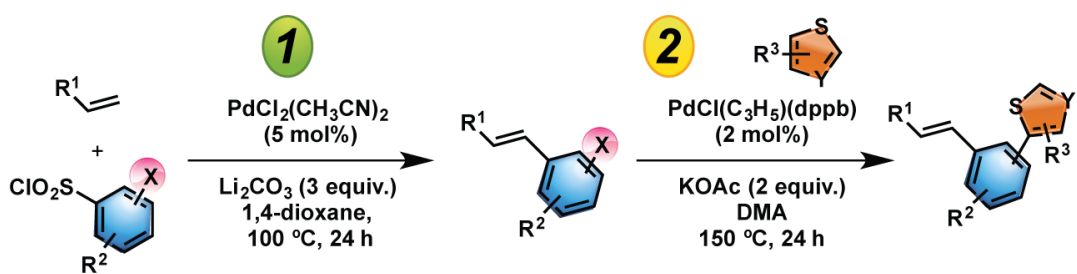

\section{Chemioselective desulfitative Heck reactions} Phosphine-free conditions, 33 examples

\section{Post-functionalizations of the $C-X$ bonds}

Abstract The palladium-catalysed desulfitative Heck type reaction of (poly)halo-substituted benzenesulfonyl chlorides with alkenes was investigated. Styrene or acrylates in the presence of bromo- or iodo-benzenesulfonyl chlorides and a phosphine-free palladium catalyst were found to afford the expected $\beta$-arylated Heck type products with complete regio- and stereo-selectivities. The reaction tolerates a variety of substituents on the halobenzenesulfonyl chloride. Moreover, no cleavage of the $\mathrm{C}-\mathrm{Br}$ and $\mathrm{C}-\mathrm{I}$ bonds was observed in the course of these reactions, allowing further transformations. Using 4-bromobenzenesulfonyl chloride as the central unit, consecutive desulfitative Heck type reaction followed by palladium-catalysed direct arylation allowed to prepare heteroarylated stilbene derivatives in only two steps.

Key words Palladium, catalysis, desulfitative Heck reaction, halobenzenesulfonyl chlorides, alkenes

\section{Introduction}

Mizoroki-Heck reaction is certainly one of the most powerful methods for the preparation of stilbene or cinnamate derivatives.1,2 For such reactions, in most cases, aryl halides were employed as the aryl source (Scheme 1); however, the reactivity of benzenesulfonyl derivatives was also studied. For example, Miura and co-workers reported in 1989 the Heck type Pd-catalysed desulfitative reaction of acrylates with benzenesulfonyl chlorides for the synthesis of 3-aryl-2-propenoates. ${ }^{3 a}$ A few years later, Vogel et al. extended these Pdcatalysed desulfitative Heck reactions to styrene and substituted acrylates. ${ }^{3 b}$ Jafarpour et al. recently reported that the reaction of methylacrylate with benzenesulfonyl chloride in the presence of $\mathrm{PdCl}_{2}$ and $\mathrm{Cu}(\mathrm{OAc})_{2}$ as catalytic system also affords the Heck type products. ${ }^{3 \mathrm{c}}$ The arylation of glycals under Pd-catalysed desulfitative Heck conditions has also been reported. ${ }^{3 \mathrm{~d}}$

$$
\begin{aligned}
& \mathrm{R} \\
& \mathrm{X}=\mathrm{Br} \text {, I, OTf, } \mathrm{SO}_{2} \mathrm{Cl} . . .
\end{aligned}
$$




\section{Scheme 1}

The synthesis of halo-substituted stilbene or cinnamate derivatives is an important field for research in organic chemistry as they give access to important building blocks for biochemists. Therefore, reaction conditions promoting Heck type reaction, tolerant to C-Halo bonds, would provide a straightforward access to halo-substituted arenes. However, although desulfitative couplings are known to tolerate both bromo and iodo substituents on benzenesulfonyl chlorides, ${ }^{4}$ surprisingly to our knowledge, only one example of desulfitative Pd-catalysed Heck-type reaction employing a bromobenzenesulfonyl chloride has been reported (Scheme 2, top). ${ }^{5 a}$ Rare examples of such Pd-catalysed reactions using a 4-bromobenzenesulfinate or bromobenzenesulfonyl hydrazides have been described (Scheme 2, middle). ${ }^{5 b, 5 d}$ A few examples of Rh- or Ru-catalysed Heck type reactions in the presence of halo-substituted arylation agents, but without cleavage of the C-halo bond, have also been reported.6,7

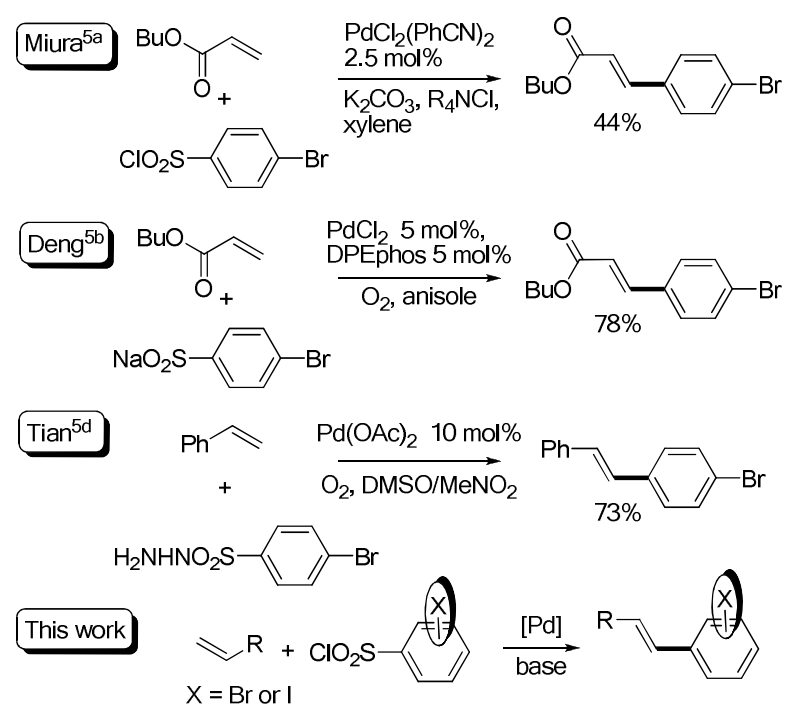

\section{Scheme 2}

As the use of (poly)halobenzenesulfonyl chlorides as reactants in Pd-catalysed reactions presents several attractive features - 1) many of them are commercially available at an affordable cost, 2) they can be easily prepared from sulfonic acids or sulphur substrates by chlorination, 3) there are generally no cleavage of the C-halo bonds in Pd-catalysed reactions - the reaction outcome using such benzenesulfonyl chlorides in Heck-type reaction needed to be investigated in more details (Scheme 2, bottom).

Herein, we report on the influence of the position of the halo-substituent on the benzenesulfonyl chlorides in the Pd-catalysed desulfitative Heck reaction. The influence of other additional substituents and the reactivity of some di- and tri-bromobenzenesulfonyl chlorides were also investigated.

\section{Results and discussion}

Based on our previous results on the Pd-catalysed desulfitative ${ }^{8}$ coupling with heteroarene derivatives, ${ }^{9,10}$ the influence of several reaction conditions, using $5 \mathrm{~mol} \% \mathrm{PdCl}_{2}(\mathrm{MeCN})_{2}$ catalyst and $\mathrm{Li}_{2} \mathrm{CO}_{3}$ as the base, on the products formation was first examined (Table 1). From 1 equiv. of 4-bromobenzenesulfonyl chloride and 1.5 equiv. of styrene at $100{ }^{\circ} \mathrm{C}$ during $24 \mathrm{~h}$, the desired Heck type product 1 was obtained in $62 \%$ yield with complete regio- and stereo-selectivity in favour of the formation of the $E$-isomer and without cleavage of the $\mathrm{C}$-Br bond (Table 1, entry 1). A lower reaction temperature of $80^{\circ} \mathrm{C}$ also gave selectively $\mathbf{1}$, but in very low yield due to a poor conversion (Table 1, entry 2). We also investigated the influence of the nature of the solvent. DMF and CPME were ineffective, as with these two solvents, 1 could not be isolated (Table 1, entries 3 and 4). The reaction performed in ethylbenzene and diethyl carbonate gave 1 in poor $22 \%$ and $12 \%$ yields, respectively (Table 1, entries 5 and 6). The use of $5 \mathrm{~mol} \% \mathrm{Pd}(\mathrm{OAc})_{2}$, afforded 1, in a slightly higher yields of 65\%; whereas, a reaction performed with $\mathrm{PdCl}_{2}$ gave 1 in $41 \%$ yield (Table 1, entries 7 and 8). When $\mathrm{K}_{2} \mathrm{CO}_{3}$ or $\mathrm{Cs}_{2} \mathrm{CO}_{3}$ were used as bases instead of $\mathrm{Li}_{2} \mathrm{CO}_{3}, \mathbf{1}$ was obtained in quite low yields (Table 1, entries 10 and 11). This difference between carbonated bases might be due to the higher solubility of $\mathrm{Cs}_{2} \mathrm{CO}_{3}$ compared with $\mathrm{Li}_{2} \mathrm{CO}_{3}$ or $\mathrm{K}_{2} \mathrm{CO}_{3}$ in dioxane. A similar trend had been previously observed in Pd-catalysed desulfitative Heck reaction or direct arylation. ${ }^{3 \mathrm{~d}, 9 \mathrm{a}, 11}$ 
Table 1. Influence of the conditions on the Pd-catalysed desulfitative reaction of styrene with 4-bromobenzenesulfonyl chloride.

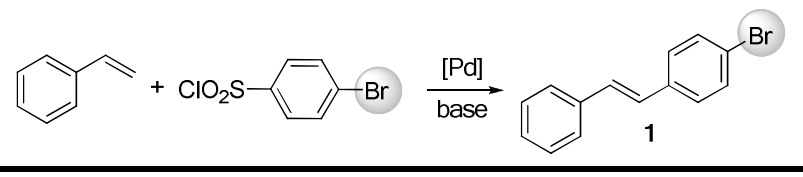

\begin{tabular}{|c|c|c|c|c|c|}
\hline Entry & Catalyst & Solvent & Base & Temp. $\left({ }^{\circ} \mathrm{C}\right)$ & Yield in $1(\%)$ \\
\hline 1 & $\mathrm{PdCl}_{2}\left(\mathrm{CH}_{3} \mathrm{CN}\right)_{2}$ & 1,4-dioxane & $\mathrm{Li}_{2} \mathrm{CO}_{3}$ & 100 & $67(62)$ \\
\hline 2 & $\mathrm{PdCl}_{2}\left(\mathrm{CH}_{3} \mathrm{CN}\right)_{2}$ & 1,4-dioxane & $\mathrm{Li}_{2} \mathrm{CO}_{3}$ & 80 & 5 \\
\hline 3 & $\mathrm{PdCl}_{2}\left(\mathrm{CH}_{3} \mathrm{CN}\right)_{2}$ & DMF & $\mathrm{Li}_{2} \mathrm{CO}_{3}$ & 150 & trace \\
\hline 4 & $\mathrm{PdCl}_{2}\left(\mathrm{CH}_{3} \mathrm{CN}\right)_{2}$ & $\mathrm{CPME}^{\mathrm{a}}$ & $\mathrm{Li}_{2} \mathrm{CO}_{3}$ & 120 & 0 \\
\hline 5 & $\mathrm{PdCl}_{2}\left(\mathrm{CH}_{3} \mathrm{CN}\right)_{2}$ & Ethylbenzene & $\mathrm{Li}_{2} \mathrm{CO}_{3}$ & 100 & 22 \\
\hline 6 & $\mathrm{PdCl}_{2}\left(\mathrm{CH}_{3} \mathrm{CN}\right)_{2}$ & Diethyl carbonate & $\mathrm{Li}_{2} \mathrm{CO}_{3}$ & 150 & 12 \\
\hline 7 & $\mathrm{Pd}(\mathrm{OAc})_{2}$ & 1,4-dioxane & $\mathrm{Li}_{2} \mathrm{CO}_{3}$ & 100 & $69(65)$ \\
\hline 8 & $\mathrm{PdCl}_{2}$ & 1,4-dioxane & $\mathrm{Li}_{2} \mathrm{CO}_{3}$ & 100 & 41 \\
\hline 10 & $\mathrm{PdCl}_{2}\left(\mathrm{CH}_{3} \mathrm{CN}\right)_{2}$ & 1,4-dioxane & $\mathrm{K}_{2} \mathrm{CO}_{3}$ & 100 & 33 \\
\hline 11 & $\mathrm{PdCl}_{2}\left(\mathrm{CH}_{3} \mathrm{CN}\right)_{2}$ & 1,4-dioxane & $\mathrm{Cs}_{2} \mathrm{CO}_{3}$ & 100 & 8 \\
\hline
\end{tabular}

Condition: [Pd] 5 mol\%, 4-bromobenzenesulfonyl chloride (1 equiv.), styrene (1.5 equiv.), $\mathrm{Li}_{2} \mathrm{CO}_{3}$ ( 3 equiv.), yield determined by GC and crude ${ }^{1} \mathrm{H}$ NMR, 24 $\mathrm{h}$, yields in parenthesis are isolated. ${ }^{a} \mathrm{CPME}$ : cyclopentyl methyl ether.

Then, the scope of the Pd-catalysed desulfitative Heck reaction of styrene with a variety of halo-substituted benzenesulfonyl chlorides was investigated (Scheme 3). A high yield of $80 \%$ in $\mathbf{2}$ was obtained for the reaction of 4-iodobenzenesulfonyl chloride with styrene. Moreover, no cleavage of the C-I bond was observed. Ortho-substituents often exhibit an important influence on Pd-catalysed reactions due to their coordination and/or steric properties. Therefore, the reactivity of 2-bromobenzenesulfonyl chloride and of a set of 2substituted 4-bromobenzenesulfonyl chlorides was investigated. 2-Bromobenzenesulfonyl chloride afforded the desired product 3 in $82 \%$ yield. Lower yields of $33 \%$ and $32 \%$ in $\mathbf{4}$ and $\mathbf{5}$ were obtained in the presence of 2-methoxy- or 2-ethyl-substituted 4bromobenzenesulfonyl chlorides. These poor yields are probably due to the formation of quite large amounts of oligomers or polymers of styrene as side-products. However, with these two substrates, the use of a larger excess of styrene ( 3 equiv.) using $\mathrm{Pd}(\mathrm{OAc})_{2}$ as catalyst allowed to increase the yield in 4 and 5 to $56 \%$ and 51\% yields, respectively. On the other hand, 4-bromo-2fluorobenzenesulfonyl chloride and more congested 4-bromo-2-(trifluoromethyl)benzenesulfonyl chloride afforded $\mathbf{6}$ and $\mathbf{7}$ in $71 \%$ and 79\% yields, respectively. From 2-bromo-4-(trifluoro)benzenesulfonyl chloride, the desired product 8 was also obtained in good yield. It should be mentioned that for all these reactions, no cleavage of the C-halo bonds was observed allowing further transformations. As both 2,5- and 3,4-dibromobenzene-1-sulfonyl chlorides can be easily prepared by reaction of 1,4- and 1,2-dibromobenzenes with chlorosulfonic acid, ${ }^{12 a}$ their reactivity for desulfitative Heck reaction was also evaluated. In both cases, the expected products $\mathbf{9}$ and $\mathbf{1 0}$ were obtained in high yields without cleavage of both C-Br bonds. Moreover, the reaction of 2,4,6-tribromobenzene-1-sulfonyl chloride with styrene was found to afford $\mathbf{1 1}$ with the three C-Br bonds untouched, but in only $12 \%$ yield. Currently, such polyhalo-substituted styrenes are generally prepared using multi-steps syntheses via Wittig reaction as the key step. ${ }^{12 b, 12 c}$

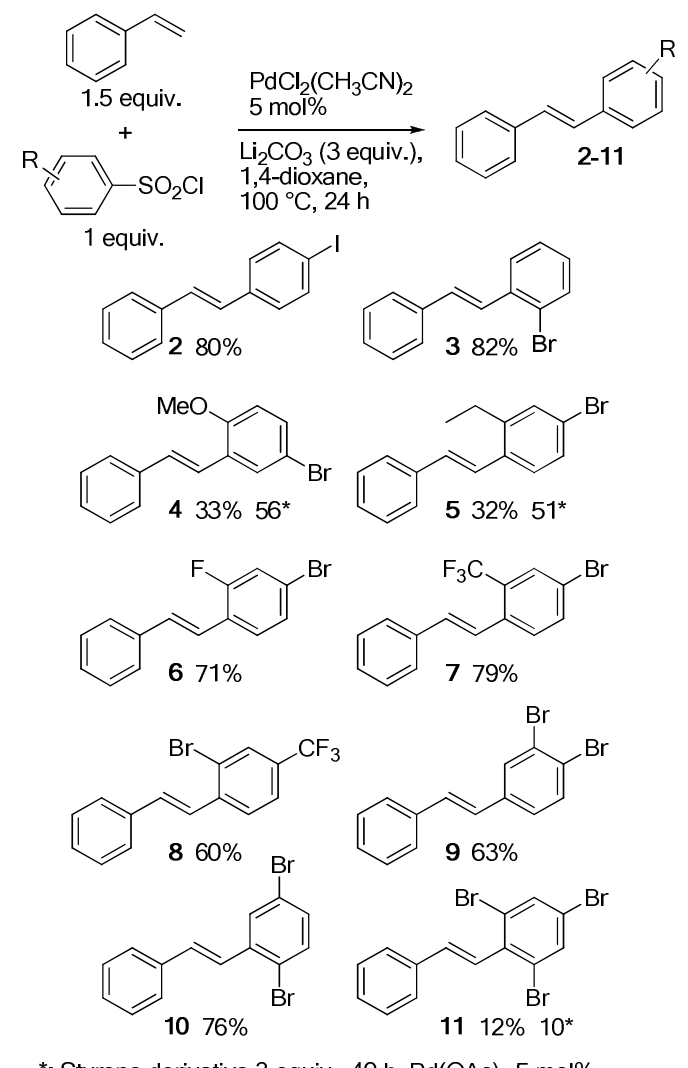

*: Styrene derivative 3 equiv., $40 \mathrm{~h}, \mathrm{Pd}(\mathrm{OAc})_{2} 5 \mathrm{~mol} \%$ 


\section{Scheme 3}

The influence of some styrene substituents on their reactivity for this reaction was then examined (Scheme 4). Lower yields than with styrene were obtained with both 4-methoxy- and 4-cyano-styrenes, as 12 and 13 were isolated in only 26\% and 51\% yields, respectively. However, a bromo substituent on styrene was tolerated allowing the synthesis of dibromostilbenes. From 4- and 3bromostyrenes and 4-bromobenzenesulfonyl chloride as reaction partner, the desired dibromostilbenes $\mathbf{1 4}$ and 15 were obtained in low to moderate yields, due to the low conversions of these two bromobenzenesulfonyl chlorides. It should be mentioned that the use of a larger excess of 3-bromostyrene using $\mathrm{Pd}(\mathrm{OAc})_{2}$ as catalyst allowed to increase the yield in $\mathbf{1 5}$ to $54 \%$. Both 4-fluorostyrene and 2,3,4,5,6-pentafluorostyrene were also successfully reacted with 4-bromobenzenesulfonyl chloride affording 16 and 17 in good yields. Again, the use of 3 equiv. of alkene with $5 \mathrm{~mol} \% \mathrm{Pd}(\mathrm{OAc})_{2}$ catalyst gave the highest yields. A moderate yield in 18 was obtained using 2vinylpyridine and 4-bromobenzenesulfonyl chloride as reaction partners. Then, we compared the reactivity of 4-bromobenzenesulfonyl chloride and 4-iodobenzenesulfonyl chloride in the presence of three styrene derivatives. Similar yields than with 4bromobenzenesulfonyl chloride were obtained in all cases. Moreover, no cleavage of the C-I bond was observed. For example, 4fluorostyrene and 2,3,4,5,6-pentafluorostyrene reacted with 4-iodobenzenesulfonyl chloride gave $\mathbf{2 0}$ and $\mathbf{2 1}$ in $\mathbf{7 3 \%}$ and $\mathbf{7 2 \%}$ yields, respectively. Again, in the presence of 4-bromostyrene, a low yield in desired product 19 was obtained.

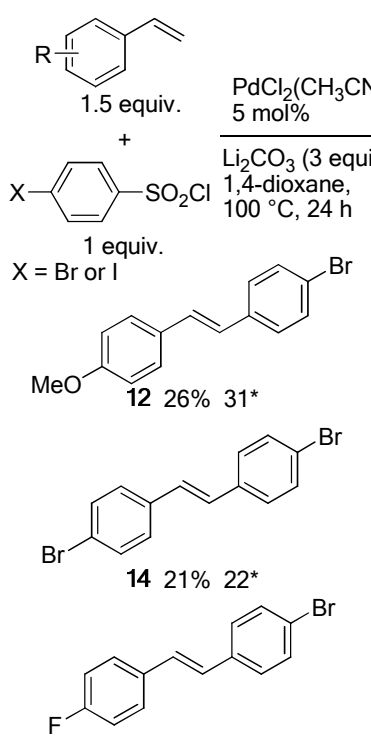

$1624 \% 61 \% *$<smiles>Brc1ccc(/C=C/c2ccccn2)cc1</smiles><smiles>Fc1ccc(/C=C/c2ccc(I)cc2)cc1</smiles>

$2073 \% *$<smiles>[R][R]1ccc(/C=C/c2ccc([X])cc2)cc1/C=C/c1ccc(C)cc1</smiles><smiles>Brc1ccc(/C=C/c2cccc(Br)c2)cc1</smiles>

15 37\% 54\%*<smiles>Fc1c(F)c(F)c(/C=C/c2ccc(Br)cc2)c(F)c1F</smiles><smiles>Brc1ccc(/C=C/c2ccc(I)cc2)cc1</smiles><smiles>[Z20]c1c(F)c(F)c(F)c(/C=C/c2ccc(I)cc2)c1F</smiles>

*: Styrene derivative 3 equiv., 40 h, $\mathrm{Pd}(\mathrm{OAc})_{2} 5 \mathrm{~mol} \%$ 


\section{Scheme 4}

The reactivity of a few other alkenes for such reactions was also investigated (Scheme 5). The reaction of $n$-butylacrylate with 4bromobenzenesulfonyl chloride gave the cinnamate derivative $\mathbf{2 2}$ in 70\% yield. Again a complete regio- and stereo-selectivity in favour of the formation of the $E$-isomer was observed. A set of substituents at C2 of 4-bromobenzenesulfonyl chloride, for reaction with $n$ butylacrylate, was also tolerated affording the bromo-substituted cinnamates $\mathbf{2 3 - 2 5}$ in $\mathbf{7 8 - 8 5 \%}$ yields. A high yield of $93 \%$ in $\mathbf{2 6}$ was also obtained for the reaction of $n$-butylacrylate with 2-bromo-4-(trifluoromethyl)benzenesulfonyl chloride. Both 2,5- and 3,4dibromobenzene-1-sulfonyl chlorides were also successfully coupled with $n$-butylacrylate affording $\mathbf{2 7}$ and $\mathbf{2 8}$ in $91 \%$ and $59 \%$ yields, respectively. The reaction of $n$-butylacrylate with 4 -iodobenzenesulfonyl chloride gave Heck type product $\mathbf{2 9}$ in $82 \%$ yield, without C-I bond cleavage. Even the electron-deficient 2-iodo-5-nitrobenzenesulfonyl chloride gave the target product $\mathbf{3 0}$ without cleavage of the very reactive $\mathrm{C}$-I bond. If terminal alkenes are reactive under these conditions in Pd-catalysed desulfitative Heck reaction, on the other hand, ethyl trans-2-butenoate ${ }^{13}$ exhibits a poor reactivity affording 31 in only $20 \%$ yield. However, the reaction was found to be fully regio- and stereo-selective. The reaction of 3,3-dimethylbut-1-ene with 4-bromobenzenesulfonyl chloride gave 32 in only $36 \%$ yield. Due to the low boiling point of 3,3-dimethylbut-1-ene, 5 equiv. of this alkene were employed for this reaction. From dimethyl(phenyl)(vinyl)silane and 4-iodobenzenesulfonyl chloride, $E$-isomer $\mathbf{3 3}$ was also stereoselectively obtained, but in low yield (31\%) is due to the partial in-situ desilylation of $\mathbf{3 0}$ affording 4-iodostyrene as side-product.
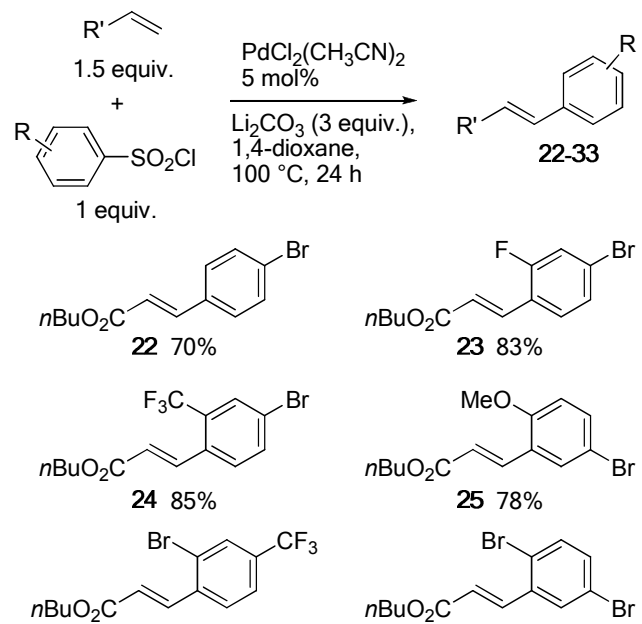

26 93\%

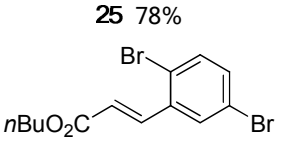

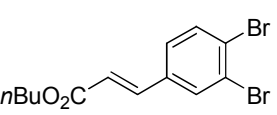

27 91\%

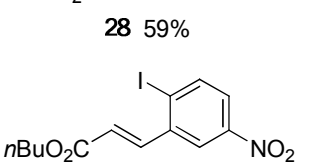

$3028 \%$<smiles>CCCCOC(=O)/C=C/c1ccc(I)cc1</smiles>

$2982 \%$<smiles>CCOC(=O)C=C(C)c1ccc(Br)cc1</smiles>

$3120 \%$<smiles>[13CH3]/C=C/c1ccc(Br)cc1</smiles>

32 36\%*

*: 5 equiv. of alkene<smiles>C[Si](C)(/C=C/c1ccc(I)cc1)c1ccccc1</smiles>

33 31\% 


\section{Scheme 5}

Although the mechanism is not yet elucidated, we assume that in the first step an oxidative addition of $\mathrm{ArSO}_{2} \mathrm{Cl}$ to $\mathrm{Pd}(\mathrm{II})$ affords a $\mathrm{Pd}(\mathrm{IV})$ species. Such oxidative addition on $\mathrm{Pd}(\mathrm{II})$ have been reported to proceed even at room temperature. ${ }^{14}$ Then, after elimination of $\mathrm{SO}_{2}$, the coordination of the alkene followed by insertion in the Pd-Ar bond might afford a Pd-CHRCH $\mathrm{Ar}_{2}$ intermediate. Then, $\beta$-H elimination followed by reductive elimination assisted by the base would produces the $\beta$-arylated alkene derivative with regeneration of a Pd(II) species.

Since one decade, Pd-catalysed direct arylation of heteroaromatics with aryl halides via a C-H bond activation has become a popular method for generating carbon-carbon bonds. ${ }^{15}$ In order to further demonstrate the synthetic potential of the halo-substituted stilbenes prepared by our method, Pd-catalysed direct arylations using 1 as aryl source was also studied (Scheme 6). Using 2 mol\% $\mathrm{PdCl}\left(\mathrm{C}_{3} \mathrm{H}_{5}\right)_{2}(\mathrm{dppb})^{16}$ catalyst in the presence of KOAc in DMA, 1 was coupled with 2-ethyl-4-methylthiazole and 2-pentylthiophene to afford 34 and 35 in 53\% and 51\% yields, respectively. In both cases, a regioselective C5-arylation of the heteroarene, without isomerisation of the stilbene double bond, was observed.

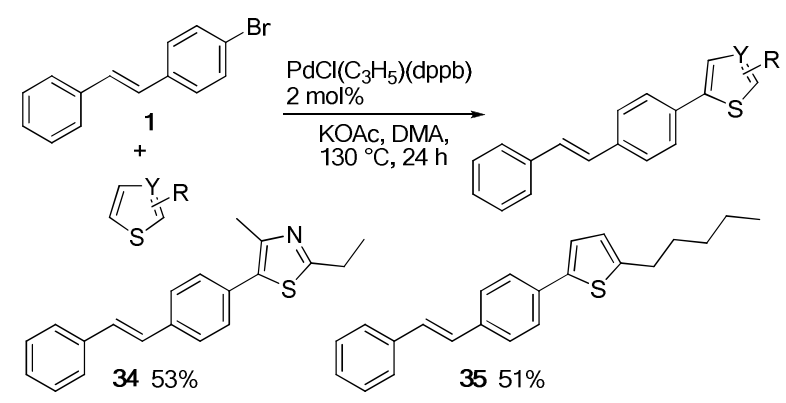

Scheme 6

\section{Conclusion}

In summary, we report here phosphine-ligand free, ammonium-salt free and oxidant-free conditions allowing desulfitative palladiumcatalysed Heck type reactions using both bromo- and iodo-substituted benzenesulfonyl chlorides, in the presence of styrenes or acrylates, as the reaction partners. In the course of these reactions, no cleavage of the benzenesulfonyl chlorides C-Br or C-I bonds was observed allowing further transformations. The reaction was found to proceed with easily accessible $\mathrm{Pd}\left(\mathrm{MeCN}_{2} \mathrm{Cl}_{2} \mathrm{Catalyst}_{\mathrm{Snd}} \mathrm{Li}_{2} \mathrm{CO}_{3}\right.$ as inexpensive base. Moreover, this procedure tolerates a variety of substituents on the halobenzenesulfonyl chlorides. Due to the wide availability of diversely functionalized (poly)halo-substituted benzenesulfonyl chlorides at an affordable cost, such simple reaction conditions (no expensive base and ligand) should be very attractive to synthetic chemists for access to (poly)halo-substituted stilbene or cinnamate derivatives, compared to more classical methods, such as Wittig reaction, which requires several steps and, in some cases, affords mixtures of stereoisomers.

\section{Experimental section}

\section{General Remarks:}

All reactions were run under argon in Schlenk tubes using vacuum lines. Dioxane analytical grade was not distilled before use. $\mathrm{Li}_{2} \mathrm{CO}_{3}$ $(>99 \%)$ was used. Commercial alkene derivatives and halobenzenesulfonyl chlorides were used without purification. The reactions were followed by GC and NMR. ${ }^{1} \mathrm{H}$ and ${ }^{13} \mathrm{C}$ spectra were recorded with a Bruker $400 \mathrm{MHz}$ spectrometer in $\mathrm{CDCl}_{3} \mathrm{Solutions} \mathrm{Chemical}$ shifts are reported in ppm relative to $\mathrm{CDCl}_{3}$ ( 7.26 for ${ }^{1} \mathrm{H}$ NMR and 77.0 for ${ }^{13} \mathrm{C}$ NMR). Flash chromatography was performed on silica gel (230-400 mesh).

General procedure for desulfitative reactions: In a typical experiment, the alkene derivative (1.5 mmol), halobenzenesulfonyl chloride derivative $(1 \mathrm{mmol}), \mathrm{Li}_{2} \mathrm{CO}_{3}(0.222 \mathrm{~g}, 3 \mathrm{mmol})$ and $\mathrm{PdCl}_{2}\left(\mathrm{MeCN}_{2}(12.9 \mathrm{mg}, 0.05 \mathrm{mmol})\right.$, were dissolved in 1,4-dioxane (2 mL) under an argon atmosphere. The reaction mixture was stirred at $100{ }^{\circ} \mathrm{C}$ for $24 \mathrm{~h}$. After evaporation of the solvent, the product was purified by silica gel column chromatography.

Preparation of the $\mathbf{P d C l}\left(\mathbf{C}_{3} \mathbf{H}_{5}\right)(\mathbf{d p p b})$ catalyst: ${ }^{16}$ An oven-dried $40 \mathrm{~mL}$ Schlenk tube equipped with a magnetic stirring bar under argon atmosphere, was charged with $\left[\mathrm{Pd}\left(\mathrm{C}_{3} \mathrm{H}_{5}\right) \mathrm{Cl}\right]_{2}(182 \mathrm{mg}, 0.5 \mathrm{mmol})$ and dppb (426 mg, $\left.1 \mathrm{mmol}\right) .10 \mathrm{~mL}$ of anhydrous dichloromethane were added, then, the solution was stirred at room temperature for twenty minutes. The solvent was removed in vacuum. The yellow powder was used without purification. ${ }^{31} \mathrm{P}$ NMR $\left(81 \mathrm{MHz}, \mathrm{CDCl}_{3}\right) \delta=19.3(\mathrm{~s})$. 
(E)-1-Bromo-4-styrylbenzene (1) ${ }^{17}$

From styrene ( $0.156 \mathrm{~g}, 1.5 \mathrm{mmol})$ and 4-bromobenzenesulfonyl chloride (0.255 g, $1 \mathrm{mmol})$, product 1 was obtained in $62 \%$ (0.160 g) yield as a white solid (mp: $141-143^{\circ} \mathrm{C}$ ).

$\left.{ }^{1} \mathrm{H} \mathrm{NMR} \mathrm{(400} \mathrm{MHz,} \mathrm{CDCl}_{3}\right) \delta 7.51(\mathrm{~d}, J=8.5 \mathrm{~Hz}, 2 \mathrm{H}), 7.48(\mathrm{~d}, J=8.5 \mathrm{~Hz}, 2 \mathrm{H}), 7.40-7.33(\mathrm{~m}, 4 \mathrm{H}), 7.28(\mathrm{t}, J=7.4 \mathrm{~Hz}, 1 \mathrm{H}), 7.10(\mathrm{~d}, J=16.4 \mathrm{~Hz}$, $1 \mathrm{H}), 7.03(\mathrm{~d}, J=16.4 \mathrm{~Hz}, 1 \mathrm{H})$.

${ }^{13} \mathrm{C}$ NMR (100 MHz, $\left.\mathrm{CDCl}_{3}\right) \delta 137.0,136.3,131.8,129.4,128.7,128.0,127.9,127.4,126.6,121.3$.

\section{(E)-1-Iodo-4-styrylbenzene (2) ${ }^{18}$}

From styrene ( $0.156 \mathrm{~g}, 1.5 \mathrm{mmol})$ and 4-iodobenzenesulfonyl chloride $(0.303 \mathrm{~g}, 1 \mathrm{mmol})$, product 2 was obtained in $80 \%$ ( $0.244 \mathrm{~g}$ ) yield as a white solid (mp: $156-159^{\circ} \mathrm{C}$ ).

${ }^{1} \mathrm{H} \mathrm{NMR}\left(400 \mathrm{MHz}, \mathrm{CDCl}_{3}\right) \delta 7.72(\mathrm{~d}, J=8.5 \mathrm{~Hz}, 2 \mathrm{H}), 7.55(\mathrm{~d}, J=8.5 \mathrm{~Hz}, 2 \mathrm{H}), 7.43-7.25(\mathrm{~m}, 5 \mathrm{H}), 7.14(\mathrm{~d}, J=16.4 \mathrm{~Hz}, 1 \mathrm{H}), 7.047 .14(\mathrm{~d}, J=$ $16.4 \mathrm{~Hz}, 1 \mathrm{H})$.

${ }^{13} \mathrm{C}$ NMR $\left(100 \mathrm{MHz}, \mathrm{CDCl}_{3}\right) \delta 137.8,137.0,136.9,129.6,128.9,128.3,128.0,127.6,126.7,91.9$.

\section{(E)-1-Bromo-2-styrylbenzene (3) ${ }^{19}$}

From styrene ( $0.156 \mathrm{~g}, 1.5 \mathrm{mmol}$ ) and 2-bromobenzenesulfonyl chloride (0.255 g, $1 \mathrm{mmol})$, product 3 was obtained in $82 \%$ (0.212 g) yield as a colourless oil.

${ }^{1} \mathrm{H} \mathrm{NMR}\left(400 \mathrm{MHz}, \mathrm{CDCl}_{3}\right) \delta 7.70(\mathrm{~d}, J=8.5 \mathrm{~Hz}, 1 \mathrm{H}), 7.65-7.58(\mathrm{~m}, 3 \mathrm{H}), 7.55(\mathrm{~d}, J=16.4 \mathrm{~Hz}, 1 \mathrm{H}), 7.46-7.32(\mathrm{~m}, 4 \mathrm{H}), 7.16(\mathrm{t}, J=7.8 \mathrm{~Hz}, 1 \mathrm{H})$, $7.09(\mathrm{~d}, J=16.4 \mathrm{~Hz}, 1 \mathrm{H})$.

${ }^{13} \mathrm{C}$ NMR $\left(100 \mathrm{MHz}, \mathrm{CDCl}_{3}\right) \delta 137.3,137.2,133.2,131.6,128.9,128.8,128.2,127.7,127.6,127.0,126.8,124.3$.

\section{(E)-4-Bromo-1-methoxy-2-styrylbenzene (4) ${ }^{20}$}

From styrene ( $0.156 \mathrm{~g}, 1.5 \mathrm{mmol})$ and 2-methoxy-5-bromobenzenesulfonyl chloride $(0.285 \mathrm{~g}, 1 \mathrm{mmol})$, product 4 was obtained in $33 \%$ $(0.095 \mathrm{~g})$ yield as a yellow oil.

${ }^{1} \mathrm{H} \mathrm{NMR}\left(400 \mathrm{MHz}, \mathrm{CDCl}_{3}\right) \delta 7.70(\mathrm{~d}, J=2.5 \mathrm{~Hz}, 1 \mathrm{H}), 7.53(\mathrm{~d}, J=8.3 \mathrm{~Hz}, 2 \mathrm{H}), 7.42-7.25(\mathrm{~m}, 5 \mathrm{H}), 7.09(\mathrm{~d}, J=16.4 \mathrm{~Hz}, 1 \mathrm{H}), 6.77(\mathrm{~d}, J=8.7 \mathrm{~Hz}$, $1 \mathrm{H}), 3.87(\mathrm{~s}, 3 \mathrm{H})$.

${ }^{13} \mathrm{C}$ NMR $\left(100 \mathrm{MHz}, \mathrm{CDCl}_{3}\right) \delta 156.0,137.5,131.1,130.4,129.0,128.8,128.7,127.9,126.8,122.2,113.4,112.7,55.9$.

\section{(E)-1-Bromo-2-ethyl-4-styrylbenzene (5)}

From styrene (0.156 g, $1.5 \mathrm{mmol}$ ) and 2-ethyl-4-bromobenzenesulfonyl chloride $(0.284 \mathrm{~g}, 1 \mathrm{mmol})$, product $\mathbf{5}$ was obtained in $32 \%$ $(0.092 \mathrm{~g})$ yield as a yellow oil.

${ }^{1} \mathrm{H} \mathrm{NMR}\left(400 \mathrm{MHz}, \mathrm{CDCl}_{3}\right) \delta 7.52(\mathrm{~d}, J=8.5 \mathrm{~Hz}, 2 \mathrm{H}), 7.49(\mathrm{~d}, J=8.5 \mathrm{~Hz}, 1 \mathrm{H}), 7.43-7.25(\mathrm{~m}, 6 \mathrm{H}), 6.99(\mathrm{~d}, J=16.4 \mathrm{~Hz}, 1 \mathrm{H}), 2.77(\mathrm{q}, J=7.6 \mathrm{~Hz}$, $2 \mathrm{H}), 1.24(\mathrm{t}, J=7.6 \mathrm{~Hz}, 3 \mathrm{H})$.

${ }^{13} \mathrm{C}$ NMR $\left(100 \mathrm{MHz}, \mathrm{CDCl}_{3}\right) \delta 144.0,137.5,134.9,131.7,130.8,129.3,128.9,128.0,127.4,126.7,125.2,121.6,26.4,15.2$.

$\mathrm{C}_{16} \mathrm{H}_{15} \mathrm{Br}$ (287.19): Calcd C 66.91, H 5.26; Found C 67.12, H 5.15.

\section{(E)-4-Bromo-2-fluoro-1-styrylbenzene (6)}

From styrene $(0.156 \mathrm{~g}, 1.5 \mathrm{mmol})$ and 2-fluoro-4-bromobenzenesulfonyl chloride $(0.273 \mathrm{~g}, 1 \mathrm{mmol})$, product 6 was obtained in $71 \%$ (0.197 g) yield as a white solid (mp: $78-80^{\circ} \mathrm{C}$ ).

${ }^{1} \mathrm{H} \mathrm{NMR}\left(400 \mathrm{MHz}, \mathrm{CDCl}_{3}\right) \delta 7.52(\mathrm{~d}, J=8.3 \mathrm{~Hz}, 2 \mathrm{H}), 7.47(\mathrm{t}, J=8.1 \mathrm{~Hz}, 1 \mathrm{H}), 7.38(\mathrm{t}, J=8.0 \mathrm{~Hz}, 2 \mathrm{H}), 7.35-7.25(\mathrm{~m}, 3 \mathrm{H}), 7.17(\mathrm{~s}, 2 \mathrm{H})$.

${ }^{13} \mathrm{C}$ NMR $\left(100 \mathrm{MHz}, \mathrm{CDCl}_{3}\right) \delta 160.1(\mathrm{~d}, J=254.1 \mathrm{~Hz}), 137.0,131.6(\mathrm{~d}, J=4.8 \mathrm{~Hz}), 128.9,128.3,128.1(\mathrm{~d}, J=4.3 \mathrm{~Hz}), 127.7(\mathrm{~d}, J=3.6 \mathrm{~Hz})$, 126.8, $124.5(\mathrm{~d}, J=12.0 \mathrm{~Hz}), 121.1(\mathrm{~d}, J=9.9 \mathrm{~Hz}), 120.0(\mathrm{~d}, J=3.4 \mathrm{~Hz}), 119.5(\mathrm{~d}, J=25.4 \mathrm{~Hz})$.

$\mathrm{C}_{14} \mathrm{H}_{10} \mathrm{BrF}$ (277.13): Calcd C 60.68, H 3.64; Found C 60.47, H 3.80.

\section{(E)-4-Bromo-1-styryl-2-(trifluoromethyl)benzene (7)}

From styrene (0.156 g, $1.5 \mathrm{mmol})$ and 2-trifluoromethyl-4-bromobenzenesulfonyl chloride $(0.323 \mathrm{~g}, 1 \mathrm{mmol})$, product 7 was obtained in $79 \%(0.258 \mathrm{~g})$ yield as a colourless oil.

${ }^{1} \mathrm{H} \mathrm{NMR}\left(400 \mathrm{MHz}, \mathrm{CDCl}_{3}\right) \delta 7.81(\mathrm{~s}, 1 \mathrm{H}), 7.66(\mathrm{~s}, 2 \mathrm{H}), 7.53(\mathrm{~d}, J=8.3 \mathrm{~Hz}, 2 \mathrm{H}), 7.45-7.35(\mathrm{~m}, 3 \mathrm{H}), 7.33(\mathrm{t}, J=8.0 \mathrm{~Hz}, 1 \mathrm{H}), 7.09(\mathrm{~d}, J=16.4$ $\mathrm{Hz}, 1 \mathrm{H})$. 
${ }^{13} \mathrm{C}$ NMR $\left(100 \mathrm{MHz}, \mathrm{CDCl}_{3}\right) \delta 136.6,135.5$ (q, $\left.J=1.7 \mathrm{~Hz}\right), 135.1,133.4,129.3$ (q, $\left.J=6.0 \mathrm{~Hz}\right), 129.1(\mathrm{q}, J=31.0 \mathrm{~Hz}), 128.9,128.7,128.6$, 127.1, $123.4(\mathrm{q}, J=274.4 \mathrm{~Hz}), 123.3(\mathrm{q}, J=2.0 \mathrm{~Hz}), 120.9$.

$\mathrm{C}_{15} \mathrm{H}_{10} \mathrm{BrF}_{3}$ (327.14): Calcd C 55.07, H 3.08; Found C 55.00, H 3.22.

\section{(E)-2-Bromo-1-styryl-4-(trifluoromethyl)benzene (8)}

From styrene $(0.156 \mathrm{~g}, 1.5 \mathrm{mmol})$ and 2-bromo-4-trifluoromethylbenzenesulfonyl chloride $(0.323 \mathrm{~g}, 1 \mathrm{mmol})$, product 8 was obtained in $60 \%(0.196 \mathrm{~g})$ yield as a colourless oil.

${ }^{1} \mathrm{H}$ NMR $\left(400 \mathrm{MHz}, \mathrm{CDCl}_{3}\right) \delta 7.86(\mathrm{~d}, J=1.7 \mathrm{~Hz}, 1 \mathrm{H}), 7.76(\mathrm{~d}, J=8.2 \mathrm{~Hz}, 1 \mathrm{H}), 7.60-7.55(\mathrm{~m}, 3 \mathrm{H}), 7.47(\mathrm{~d}, J=16.4 \mathrm{~Hz}, 1 \mathrm{H}), 7.39(\mathrm{t}, J=8.0 \mathrm{~Hz}$, $2 \mathrm{H}), 7.35(\mathrm{t}, J=8.0 \mathrm{~Hz}, 1 \mathrm{H}), 7.13(\mathrm{~d}, J=16.4 \mathrm{~Hz}, 1 \mathrm{H})$.

${ }^{13} \mathrm{C}$ NMR $\left(100 \mathrm{MHz}, \mathrm{CDCl}_{3}\right) \delta 140.8,136.5,133.9,130.5(\mathrm{q}, J=33.1 \mathrm{~Hz}), 130.2(\mathrm{q}, J=3.0 \mathrm{~Hz}), 128.9,128.8,127.2,126.9,126.2,124.5(\mathrm{q}, J=$ $3.7 \mathrm{~Hz}), 124.0,123.2(\mathrm{q}, J=272.4 \mathrm{~Hz})$.

$\mathrm{C}_{15} \mathrm{H}_{10} \mathrm{BrF}_{3}$ (327.14): Calcd C 55.07, H 3.08; Found C 55.30, H 3.07.

\section{(E)-1,2-Dibromo-4-styrylbenzene (9)}

From styrene ( $0.156 \mathrm{~g}, 1.5 \mathrm{mmol}$ ) and 3,4-dibromobenzene-1-sulfonyl chloride ( $0.334 \mathrm{~g}, 1 \mathrm{mmol})$, product 9 was obtained in $63 \%$ ( 0.213 g) yield as a yellow oil.

${ }^{1} \mathrm{H} \mathrm{NMR}\left(400 \mathrm{MHz}, \mathrm{CDCl}_{3}\right) \delta 7.76(\mathrm{~d}, J=2.1 \mathrm{~Hz}, 1 \mathrm{H}), 7.57(\mathrm{~d}, J=8.3 \mathrm{~Hz}, 1 \mathrm{H}), 7.50(\mathrm{~d}, J=8.5 \mathrm{~Hz}, 2 \mathrm{H}), 7.37(\mathrm{t}, J=7.8 \mathrm{~Hz}, 2 \mathrm{H}), 7.35-7.25(\mathrm{~m}$, $2 \mathrm{H}), 7.11(\mathrm{~d}, J=16.4 \mathrm{~Hz}, 1 \mathrm{H}), 6.97(\mathrm{~d}, J=16.4 \mathrm{~Hz}, 1 \mathrm{H})$.

${ }^{13} \mathrm{C}$ NMR $\left(100 \mathrm{MHz}, \mathrm{CDCl}_{3}\right) \delta 138.3,136.6,133.8,131.4,130.8,128.9,128.4,126.8,126.5,126.1,125.2,123.3$.

$\mathrm{C}_{14} \mathrm{H}_{10} \mathrm{Br}_{2}$ (338.04): Calcd C 49.74, H 2.98; Found C 49.89, H 3.12.

\section{(E)-1,4-Dibromo-2-styrylbenzene (10)}

From styrene ( $0.156 \mathrm{~g}, 1.5 \mathrm{mmol})$ and 2,5-dibromobenzene-1-sulfonyl chloride (0.334 g, $1 \mathrm{mmol})$, product 10 was obtained in $76 \%$ $(0.257 \mathrm{~g})$ yield as a yellow solid (mp: $\left.62-64^{\circ} \mathrm{C}\right)$.

${ }^{1} \mathrm{H}$ NMR (400 MHz, $\left.\mathrm{CDCl}_{3}\right) \delta 7.79(\mathrm{~d}, J=2.3 \mathrm{~Hz}, 1 \mathrm{H}), 7.55(\mathrm{~d}, J=8.3 \mathrm{~Hz}, 1 \mathrm{H}), 7.46-7.20(\mathrm{~m}, 7 \mathrm{H}), 7.04(\mathrm{~d}, J=16.4 \mathrm{~Hz}, 1 \mathrm{H})$.

${ }^{13} \mathrm{C}$ NMR $\left(100 \mathrm{MHz}, \mathrm{CDCl}_{3}\right) \delta 139.2,136.7,134.5,132.8,131.6,129.6,128.9,128.6,127.1,126.3,122.7,121.6$.

$\mathrm{C}_{14} \mathrm{H}_{10} \mathrm{Br}_{2}$ (338.04): Calcd C 49.74, H 2.98; Found C 49.47, H 3.18.

\section{(E)-1,3,5-Tribromo-2-styrylbenzene (11)}

From styrene $(0.156 \mathrm{~g}, 1.5 \mathrm{mmol})$ and 2,4,6-tribromobenzene-1-sulfonyl chloride $(0.620 \mathrm{~g}, 1 \mathrm{mmol})$, product $\mathbf{1 1}$ was obtained in $12 \%$ $(0.050 \mathrm{~g})$ yield as a white solid (mp: $\left.80-82^{\circ} \mathrm{C}\right)$.

${ }^{1} \mathrm{H} \mathrm{NMR}\left(400 \mathrm{MHz}, \mathrm{CDCl}_{3}\right) \delta 7.76(\mathrm{~s}, 2 \mathrm{H}), 7.54(\mathrm{~d}, J=8.0 \mathrm{~Hz}, 2 \mathrm{H}), 7.39(\mathrm{t}, J=8.0 \mathrm{~Hz}, 2 \mathrm{H}), 7.35(\mathrm{t}, J=8.0 \mathrm{~Hz}, 1 \mathrm{H}), 6.97(\mathrm{~d}, J=16.4 \mathrm{~Hz}, 1 \mathrm{H})$, $6.92(\mathrm{~d}, J=16.4 \mathrm{~Hz}, 1 \mathrm{H})$.

${ }^{13} \mathrm{C}$ NMR $\left(100 \mathrm{MHz}, \mathrm{CDCl}_{3}\right) \delta 137.5,137.4,136.3,134.9,128.9,128.7,126.9,126.1,124.5,121.0$.

$\mathrm{C}_{14} \mathrm{H}_{9} \mathrm{Br}_{3}$ (416.93): Calcd C 40.33, H 2.18; Found C 40.54, H 2.01.

\section{(E)-1-Bromo-4-(4-methoxystyryl)benzene (12) ${ }^{21}$}

From 4-methoxystyrene ( $0.201 \mathrm{~g}, 1.5 \mathrm{mmol})$ and 4-bromobenzenesulfonyl chloride $(0.255 \mathrm{~g}, 1 \mathrm{mmol})$, product 12 was obtained in $26 \%$ $(0.075 \mathrm{~g})$ yield as a white solid (mp: $\left.207-209^{\circ} \mathrm{C}\right)$.

${ }^{1} \mathrm{H} \mathrm{NMR}\left(400 \mathrm{MHz}, \mathrm{CDCl}_{3}\right) \delta 7.47(\mathrm{~d}, J=8.0 \mathrm{~Hz}, 2 \mathrm{H}), 7.43(\mathrm{~d}, J=8.0 \mathrm{~Hz}, 2 \mathrm{H}), 7.34(\mathrm{~d}, J=8.0 \mathrm{~Hz}, 2 \mathrm{H}), 7.05(\mathrm{~d}, J=16.4 \mathrm{~Hz}, 1 \mathrm{H}), 6.93-6.87(\mathrm{~m}$, $3 \mathrm{H}), 3.83(\mathrm{~s}, 3 \mathrm{H})$.

${ }^{13} \mathrm{C}$ NMR $\left(100 \mathrm{MHz}, \mathrm{CDCl}_{3}\right) \delta 159.7,136.8,131.9,129.9,129.1,127.9,127.8,125.4,120.9,114.3,55.5$.

\section{(E)-4-(4-Bromostyryl)benzonitrile (13) ${ }^{22}$}

From 4-cyanostyrene $(0.194 \mathrm{~g}, 1.5 \mathrm{mmol})$ and 4-bromobenzenesulfonyl chloride $(0.255 \mathrm{~g}, 1 \mathrm{mmol})$, product 13 was obtained in $51 \%$ $(0.145 \mathrm{~g})$ yield as a yellow solid (mp: $\left.192-194^{\circ} \mathrm{C}\right)$.

${ }^{1} \mathrm{H} \mathrm{NMR}\left(400 \mathrm{MHz}, \mathrm{CDCl}_{3}\right) \delta 7.61(\mathrm{~d}, J=8.0 \mathrm{~Hz}, 2 \mathrm{H}), 7.57(\mathrm{~d}, J=8.0 \mathrm{~Hz}, 2 \mathrm{H}), 7.51(\mathrm{~d}, J=8.0 \mathrm{~Hz}, 2 \mathrm{H}), 7.39(\mathrm{~d}, J=8.0 \mathrm{~Hz}, 2 \mathrm{H}), 7.15(\mathrm{~d}, J=16.4$ $\mathrm{Hz}, 1 \mathrm{H}), 7.07(\mathrm{~d}, J=16.4 \mathrm{~Hz}, 1 \mathrm{H})$. 


\section{(E)-1,2-Bis(4-bromophenyl)ethane (14) ${ }^{23}$}

From 4-bromostyrene $(0.183 \mathrm{~g}, 1.5 \mathrm{mmol})$ and 4-bromobenzenesulfonyl chloride $(0.255 \mathrm{~g}, 1 \mathrm{mmol})$, product $\mathbf{1 4}$ was obtained in $21 \%$ $(0.071 \mathrm{~g})$ yield as a white solid (mp: $\left.216-219^{\circ} \mathrm{C}\right)$.

${ }^{1} \mathrm{H} \mathrm{NMR}\left(400 \mathrm{MHz}, \mathrm{CDCl}_{3}\right) \delta 7.48(\mathrm{~d}, J=8.4 \mathrm{~Hz}, 4 \mathrm{H}), 7.36(\mathrm{~d}, J=8.4 \mathrm{~Hz}, 4 \mathrm{H}), 7.02(\mathrm{~s}, 2 \mathrm{H})$.

${ }^{13} \mathrm{C}$ NMR $\left(100 \mathrm{MHz}, \mathrm{CDCl}_{3}\right) \delta 136.1,132.0,128.3,128.2,121.8$.

\section{(E)-1-Bromo-3-(4-bromostyryl)benzene (15) ${ }^{24}$}

From 3-bromostyrene ( $0.366 \mathrm{~g}, 1.5 \mathrm{mmol})$ and 4-bromobenzenesulfonyl chloride $(0.255 \mathrm{~g}, 1 \mathrm{mmol})$, product $\mathbf{1 5}$ was obtained in $54 \%$ (0.182 g) yield as a white solid (mp: $97-100{ }^{\circ} \mathrm{C}$ ).

${ }^{1} \mathrm{H} \mathrm{NMR}\left(400 \mathrm{MHz}, \mathrm{CDCl}_{3}\right) \delta 7.65(\mathrm{~s}, 1 \mathrm{H}), 7.49(\mathrm{~d}, J=8.4 \mathrm{~Hz}, 2 \mathrm{H}), 7.43-7.38(\mathrm{~m}, 2 \mathrm{H}), 7.36(\mathrm{~d}, J=8.4 \mathrm{~Hz}, 2 \mathrm{H}), 7.22(\mathrm{t}, J=8.0 \mathrm{~Hz}, 1 \mathrm{H}), 7.03(\mathrm{~d}$, $J=16.4 \mathrm{~Hz}, 1 \mathrm{H}), 6.99(\mathrm{~d}, J=16.4 \mathrm{~Hz}, 1 \mathrm{H})$.

${ }^{13} \mathrm{C}$ NMR $\left(100 \mathrm{MHz}, \mathrm{CDCl}_{3}\right) \delta 139.3,135.9,132.0,130.8,130.3,129.4,129.0,128.2,127.9,125.4,123.1,121.9$.

\section{(E)-1-Bromo-4-(4-fluorostyryl)benzene (16) ${ }^{25}$}

From 4-fluorostyrene $(0.244 \mathrm{~g}, 3 \mathrm{mmol})$ and 4-bromobenzenesulfonyl chloride (0.255 g, 1 mmol), product 16 was obtained in $61 \%$ $(0.169 \mathrm{~g})$ yield as a white solid (mp: $\left.138-140^{\circ} \mathrm{C}\right)$.

${ }^{1} \mathrm{H} \mathrm{NMR}\left(400 \mathrm{MHz}, \mathrm{CDCl}_{3}\right) \delta 7.53-7.45(\mathrm{~m}, 4 \mathrm{H}), 7.35(\mathrm{~d}, J=8.4 \mathrm{~Hz}, 2 \mathrm{H}), 7.09-7.02(\mathrm{~m}, 3 \mathrm{H}), 6.92(\mathrm{~d}, J=16.4 \mathrm{~Hz}, 1 \mathrm{H})$.

${ }^{13} \mathrm{C}$ NMR $\left(100 \mathrm{MHz}, \mathrm{CDCl}_{3}\right) \delta 162.6(\mathrm{~d}, J=257.5 \mathrm{~Hz}), 136.3,133.3,132.0,128.4,128.2(\mathrm{~d}, J=8.0 \mathrm{~Hz}), 128.0,127.4(\mathrm{~d}, J=2.4 \mathrm{~Hz}), 121.5$, $115.8(\mathrm{~d}, J=21.9 \mathrm{~Hz})$.

\section{(E)-1-(4-bromostyryl)-2,3,4,5,6-pentafluorobenzene (17) ${ }^{23}$}

From 2,3,4,5,6-pentafluorostyrene $(0.582 \mathrm{~g}, 3 \mathrm{mmol})$ and 4-bromobenzenesulfonyl chloride $(0.255 \mathrm{~g}, 1 \mathrm{mmol})$, product 17 was obtained in $74 \%(0.258 \mathrm{~g})$ yield as a white solid (mp: $\left.104-106^{\circ} \mathrm{C}\right)$.

${ }^{1} \mathrm{H}$ NMR (400 MHz, $\left.\mathrm{CDCl}_{3}\right) \delta 7.53(\mathrm{~d}, J=8.4 \mathrm{~Hz}, 2 \mathrm{H}), 7.40(\mathrm{~d}, J=8.4 \mathrm{~Hz}, 2 \mathrm{H}), 7.37(\mathrm{~d}, J=16.8 \mathrm{~Hz}, 1 \mathrm{H}), 6.97(\mathrm{~d}, J=16.8 \mathrm{~Hz}, 1 \mathrm{H})$.

${ }^{13} \mathrm{C}$ NMR $\left(100 \mathrm{MHz}, \mathrm{CDCl}_{3}\right) \delta 146.9(\mathrm{dm}, J=250.0 \mathrm{~Hz}), 140.0(\mathrm{dm}, J=250.0 \mathrm{~Hz}), 137.2(\mathrm{dm}, J=250.0 \mathrm{~Hz}), 138.6(\mathrm{~m}), 137.7,132.0,128.3$, 123.0, $113.4(\mathrm{~m}), 112.0(\mathrm{t}, J=13.6 \mathrm{~Hz})$.

\section{(E)-2-(4-Bromostyryl)pyridine (18) ${ }^{26}$}

From 2-vinylpyridine (0.158 g, $1.5 \mathrm{mmol})$ and 4-bromobenzenesulfonyl chloride $(0.255 \mathrm{~g}, 1 \mathrm{mmol})$, product $\mathbf{1 8}$ was obtained in $31 \%$ (0.081 g) yield as a brown solid (mp: $\left.106-108^{\circ} \mathrm{C}\right)$.

${ }^{1} \mathrm{H} \mathrm{NMR}\left(400 \mathrm{MHz}, \mathrm{CDCl}_{3}\right) \delta 8.61(\mathrm{~d}, J=5.1 \mathrm{~Hz}, 1 \mathrm{H}), 7.82(\mathrm{~d}, J=8.1 \mathrm{~Hz}, 2 \mathrm{H}), 7.74(\mathrm{td}, J=7.7,1.6 \mathrm{~Hz}, 1 \mathrm{H}), 7.68(\mathrm{~d}, J=8.1 \mathrm{~Hz}, 2 \mathrm{H}), 7.64(\mathrm{~d}, J=$ $14.9 \mathrm{~Hz}, 1 \mathrm{H}), 7.42$ (d, $J=14.9 \mathrm{~Hz}, 1 \mathrm{H}), 7.40$ (d, $J=7.7 \mathrm{~Hz}, 1 \mathrm{H}), 7.28(\mathrm{dd}, J=7.7,5.1 \mathrm{~Hz}), 1 \mathrm{H})$.

${ }^{13} \mathrm{C}$ NMR $\left(100 \mathrm{MHz}, \mathrm{CDCl}_{3}\right) \delta 150.9,150.5,141.2,139.5,137.2,132.8,131.5,129.6,129.0,125.7,125.3$.

\section{(E)-1-Bromo-4-(4-iodostyryl)benzene (19) ${ }^{27}$}

From 4-bromostyrene ( $0.366 \mathrm{~g}, 3 \mathrm{mmol}$ ) and 4-iodobenzenesulfonyl chloride (0.303 g, $1 \mathrm{mmol}$ ), product 19 was obtained in $20 \%$ (0.077 g) yield as a white solid (mp: $240-242^{\circ} \mathrm{C}$ ).

${ }^{1} \mathrm{H} \mathrm{NMR}\left(400 \mathrm{MHz}, \mathrm{CDCl}_{3}\right) \delta 7.67(\mathrm{~d}, J=8.2 \mathrm{~Hz}, 2 \mathrm{H}), 7.47(\mathrm{~d}, J=8.2 \mathrm{~Hz}, 2 \mathrm{H}), 7.36(\mathrm{~d}, J=8.2 \mathrm{~Hz}, 2 \mathrm{H}), 7.23(\mathrm{~d}, J=8.2 \mathrm{~Hz}, 2 \mathrm{H}), 7.03(\mathrm{~d}, J=16.3$ $\mathrm{Hz}, 1 \mathrm{H}), 6.97(\mathrm{~d}, J=16.3 \mathrm{~Hz}, 1 \mathrm{H})$.

\section{(E)-1-Fluoro-4-(4-iodostyryl)benzene (20) ${ }^{28}$}

From 4-fluorostyrene ( $0.244 \mathrm{~g}, 3 \mathrm{mmol}$ ) and 4-iodobenzenesulfonyl chloride ( $0.303 \mathrm{~g}, 1 \mathrm{mmol}$ ), product 20 was obtained in $73 \%$ (0.236 g) yield as a white solid (mp: $165-167^{\circ} \mathrm{C}$ ).

${ }^{1} \mathrm{H}$ NMR (400 MHz, $\left.\mathrm{CDCl}_{3}\right) \delta 7.68(\mathrm{~d}, J=8.3 \mathrm{~Hz}, 2 \mathrm{H}), 7.47(\mathrm{dd}, J=8.6,5.5 \mathrm{~Hz}, 2 \mathrm{H}), 7.23(\mathrm{~d}, J=8.3 \mathrm{~Hz}, 2 \mathrm{H}), 7.10-7.03(\mathrm{~m}, 3 \mathrm{H}), 6.92(\mathrm{~d}, J=$ $16.4 \mathrm{~Hz}, 1 \mathrm{H})$. 


\section{(E)-1,2,3,4,5-Pentafluoro-6-(4-iodostyryl)benzene (21)}

From 2,3,4,5,6-pentafluorostyrene ( $0.582 \mathrm{~g}, 3 \mathrm{mmol})$ and 4-iodobenzenesulfonyl chloride ( $0.303 \mathrm{~g}, 1 \mathrm{mmol})$, product 21 was obtained in $72 \%(0.285 \mathrm{~g})$ yield as a white solid (mp: $\left.107-110^{\circ} \mathrm{C}\right)$.

${ }^{1} \mathrm{H} \mathrm{NMR}\left(400 \mathrm{MHz}, \mathrm{CDCl}_{3}\right) \delta 7.71(\mathrm{~d}, J=8.2 \mathrm{~Hz}, 2 \mathrm{H}), 7.34(\mathrm{~d}, J=16.8 \mathrm{~Hz}, 1 \mathrm{H}), 7.25(\mathrm{~d}, J=8.2 \mathrm{~Hz}, 2 \mathrm{H}), 6.97(\mathrm{~d}, J=16.8 \mathrm{~Hz}, 1 \mathrm{H})$.

${ }^{13} \mathrm{C}$ NMR (100 MHz, $\left.\mathrm{CDCl}_{3}\right) \delta 144.9(\mathrm{dm}, J=250.0 \mathrm{~Hz}), 140.1(\mathrm{dm}, J=250.0 \mathrm{~Hz}), 138.2,137.8(\mathrm{dm}, J=250.0 \mathrm{~Hz}), 136.1(\mathrm{~m}), 128.6,113.5$, $112.3(\mathrm{t}, J=13.6 \mathrm{~Hz}), 94.8$.

$\mathrm{C}_{14} \mathrm{H}_{6} \mathrm{~F}_{5} \mathrm{I}$ (396.09): Calcd C 42.45, H 1.53; Found C 42.55, H 1.41.

(E)-Butyl 3-(4-bromophenyl)acrylate (22) ${ }^{7}$

From $n$-butylacrylate $(0.192 \mathrm{~g}, 1.5 \mathrm{mmol})$ and 4-bromobenzenesulfonyl chloride $(0.255 \mathrm{~g}, 1 \mathrm{mmol})$, product 22 was obtained in $70 \%$ (0.198 g) yield as a white solid (mp: $37-39^{\circ} \mathrm{C}$ ).

${ }^{1} \mathrm{H} \mathrm{NMR}\left(400 \mathrm{MHz}, \mathrm{CDCl}_{3}\right) \delta 7.60(\mathrm{~d}, J=16.0 \mathrm{~Hz}, 1 \mathrm{H}), 7.50(\mathrm{~d}, J=8.3 \mathrm{~Hz}, 2 \mathrm{H}), 7.37(\mathrm{~d}, J=8.3 \mathrm{~Hz}, 2 \mathrm{H}), 6.42(\mathrm{~d}, J=16.0 \mathrm{~Hz}, 1 \mathrm{H}), 4.20(\mathrm{t}, J=$ $7.6 \mathrm{~Hz}, 2 \mathrm{H}$ ), 1.68 (quint., $J=7.6 \mathrm{~Hz}, 2 \mathrm{H}$ ), 1.41 (sext., $J=7.6 \mathrm{~Hz}, 2 \mathrm{H}$ ), 0.95 (t, $J=7.6 \mathrm{~Hz}, 3 \mathrm{H}$ ).

${ }^{13} \mathrm{C}$ NMR (100 MHz, $\left.\mathrm{CDCl}_{3}\right) \delta 166.9,143.2,133.5,132.2,129.5,124.5,119.1,64.7,30.9,19.3,13.9$.

\section{(E)-Butyl 3-(4-bromo-2-fluorophenyl)acrylate (23)}

From $n$-butylacrylate $(0.192 \mathrm{~g}, 1.5 \mathrm{mmol})$ and 2 -fluoro-4-bromobenzene-1-sulfonyl chloride $(0.273 \mathrm{~g}, 1 \mathrm{mmol})$, product 23 was obtained in $83 \%(0.250 \mathrm{~g})$ yield as a white solid ( $\left.\mathrm{mp}: 36-38^{\circ} \mathrm{C}\right)$.

${ }^{1} \mathrm{H} \mathrm{NMR}\left(400 \mathrm{MHz}, \mathrm{CDCl}_{3}\right) \delta 7.72(\mathrm{~d}, J=16.0 \mathrm{~Hz}, 1 \mathrm{H}), 7.41(\mathrm{t}, J=9.0 \mathrm{~Hz}, 1 \mathrm{H}), 7.33-7.26(\mathrm{~m}, 2 \mathrm{H}), 6.52(\mathrm{~d}, J=16.0 \mathrm{~Hz}, 1 \mathrm{H}), 4.21(\mathrm{t}, J=7.6 \mathrm{~Hz}$, 2H), 1.68 (quint., $J=7.6 \mathrm{~Hz}, 2 \mathrm{H}$ ), 1.41 (sext., $J=7.6 \mathrm{~Hz}, 2 \mathrm{H}$ ), 0.95 (t, $J=7.6 \mathrm{~Hz}, 3 \mathrm{H}$ ).

${ }^{13} \mathrm{C}$ NMR $\left(100 \mathrm{MHz}, \mathrm{CDCl}_{3}\right) \delta 166.8,160.9(\mathrm{~d}, J=258.4 \mathrm{~Hz}), 136.1$ (d, $\left.J=2.5 \mathrm{~Hz}\right), 130.0(\mathrm{~d}, J=3.6 \mathrm{~Hz}), 128.0(\mathrm{~d}, J=3.7 \mathrm{~Hz}), 124.4(\mathrm{~d}, J=9.7$ $\mathrm{Hz}), 121.8(\mathrm{~d}, J=11.8 \mathrm{~Hz}), 121.5(\mathrm{~d}, J=6.6 \mathrm{~Hz}), 120.1(\mathrm{~d}, J=25.3 \mathrm{~Hz}), 64.8,30.9,19.3,13.8$.

$\mathrm{C}_{13} \mathrm{H}_{14} \mathrm{BrFO}_{2}$ (301.15): Calcd C 51.85, H 4.69; Found C 51.59, H 4.87.

\section{(E)-Butyl 3-(4-bromo-2-(trifluoromethyl)phenyl)acrylate (24)}

From $n$-butylacrylate $(0.192 \mathrm{~g}, 1.5 \mathrm{mmol})$ and 2-trifluoromethyl-4-bromobenzenesulfonyl chloride (0.323 g, 1 mmol), product 24 was obtained in $85 \%$ (0.298 g) yield as a colourless oil.

${ }^{1} \mathrm{H} \mathrm{NMR}\left(400 \mathrm{MHz}, \mathrm{CDCl}_{3}\right) \delta 7.95(\mathrm{~d}, J=16.0 \mathrm{~Hz}, 1 \mathrm{H}), 7.83(\mathrm{~s}, 1 \mathrm{H}), 7.69(\mathrm{~d}, J=8.4 \mathrm{~Hz}, 1 \mathrm{H}), 7.57(\mathrm{~d}, J=8.4 \mathrm{~Hz}, 1 \mathrm{H}), 6.40(\mathrm{~d}, J=16.0 \mathrm{~Hz}, 1 \mathrm{H})$, $4.22(\mathrm{t}, J=7.6 \mathrm{~Hz}, 2 \mathrm{H}$ ), 1.68 (quint., $J=7.6 \mathrm{~Hz}, 2 \mathrm{H}$ ), 1.41 (sext., $J=7.6 \mathrm{~Hz}, 2 \mathrm{H}), 0.95$ (t, $J=7.6 \mathrm{~Hz}, 3 \mathrm{H}$ ).

${ }^{13} \mathrm{C}$ NMR $\left(100 \mathrm{MHz}, \mathrm{CDCl}_{3}\right) \delta 166.0,138.9,135.4,132.5,130.4(\mathrm{q}, J=31.1 \mathrm{~Hz}), 129.6(\mathrm{q}, J=5.8 \mathrm{~Hz}), 129.5,123.8,123.3,123.0(\mathrm{q}, J=274.7$ $\mathrm{Hz}), 65.0,30.8,19.3,13.9$.

$\mathrm{C}_{14} \mathrm{H}_{14} \mathrm{BrF}_{3} \mathrm{O}_{2}$ (351.16): Calcd C 47.88, H 4.02; Found C 47.79, H 4.14.

\section{(E)-Butyl 3-(5-bromo-2-methoxyphenyl)acrylate (25)}

From $n$-butylacrylate $(0.192 \mathrm{~g}, 1.5 \mathrm{mmol})$ and 5-bromo-2-methoxybenzene-1-sulfonyl chloride $(0.285 \mathrm{~g}, 1 \mathrm{mmol})$, product 25 was obtained in $78 \%(0.244 \mathrm{~g})$ yield as a white solid (mp: $\left.60-62^{\circ} \mathrm{C}\right)$.

${ }^{1} \mathrm{H}$ NMR $\left(400 \mathrm{MHz}, \mathrm{CDCl}_{3}\right) \delta 7.88(\mathrm{~d}, J=16.0 \mathrm{~Hz}, 1 \mathrm{H}), 7.60(\mathrm{~d}, J=2.5 \mathrm{~Hz}, 1 \mathrm{H}), 7.41(\mathrm{dd}, J=8.8,2.5 \mathrm{~Hz}, 1 \mathrm{H}), 6.78(\mathrm{~d}, J=8.8 \mathrm{~Hz}, 1 \mathrm{H}), 6.48(\mathrm{~d}, J$ $=16.0 \mathrm{~Hz}, 1 \mathrm{H}$ ), $4.20(\mathrm{t}, J=7.6 \mathrm{~Hz}, 2 \mathrm{H}), 3.86(\mathrm{~s}, 3 \mathrm{H}), 1.68$ (quint., $J=7.6 \mathrm{~Hz}, 2 \mathrm{H}), 1.41$ (sext., $J=7.6 \mathrm{~Hz}, 2 \mathrm{H}), 0.96(\mathrm{t}, J=7.6 \mathrm{~Hz}, 3 \mathrm{H})$.

${ }^{13} \mathrm{C}$ NMR $\left(100 \mathrm{MHz}, \mathrm{CDCl}_{3}\right) \delta 167.2,157.4,138.4,133.8,131.2,125.6,120.2,113.1,113.0,64.5,55.9,30.9,19.3,13.9$.

$\mathrm{C}_{14} \mathrm{H}_{17} \mathrm{BrO}_{3}$ (313.19): Calcd C 53.69, H 5.47; Found C 53.48, H 5.38.

\section{(E)-Butyl 3-(2-bromo-4-(trifluoromethyl)phenyl)acrylate (26)}

From $n$-butylacrylate $(0.192 \mathrm{~g}, 1.5 \mathrm{mmol})$ and 2-bromo-4-trifluoromethylbenzenesulfonyl chloride (0.323 g, 1 mmol), product 26 was obtained in $93 \%$ ( $0.326 \mathrm{~g}$ ) yield as a colourless oil.

${ }^{1} \mathrm{H} \mathrm{NMR}\left(400 \mathrm{MHz}, \mathrm{CDCl}_{3}\right) \delta 7.99(\mathrm{~d}, J=16.0 \mathrm{~Hz}, 1 \mathrm{H}), 7.87(\mathrm{~s}, 1 \mathrm{H}), 7.69(\mathrm{~d}, J=8.4 \mathrm{~Hz}, 1 \mathrm{H}), 7.54(\mathrm{~d}, J=8.4 \mathrm{~Hz}, 1 \mathrm{H}), 6.45(\mathrm{~d}, J=16.0 \mathrm{~Hz}, 1 \mathrm{H})$, $4.24(\mathrm{t}, J=7.6 \mathrm{~Hz}, 2 \mathrm{H}$ ), 1.68 (quint., $J=7.6 \mathrm{~Hz}, 2 \mathrm{H}$ ), 1.41 (sext., $J=7.6 \mathrm{~Hz}, 2 \mathrm{H}$ ), $0.95(\mathrm{t}, J=7.6 \mathrm{~Hz}, 3 \mathrm{H}$ ).

${ }^{13} \mathrm{C}$ NMR $\left(100 \mathrm{MHz}, \mathrm{CDCl}_{3}\right) \delta 166.1,141.6,138.3,132.9(\mathrm{q}, J=33.5 \mathrm{~Hz}), 130.5(\mathrm{q}, J=3.0 \mathrm{~Hz}), 128.2,125.2,124.6(\mathrm{q}, J=3.7 \mathrm{~Hz}), 123.6$, $123.0(\mathrm{q}, J=272.7 \mathrm{~Hz}), 65.0,30.8,19.3,13.9$.

$\mathrm{C}_{14} \mathrm{H}_{14} \mathrm{BrF}_{3} \mathrm{O}_{2}$ (351.16): Calcd C 47.88, H 4.02; Found C 47.98, H 4.19. 


\section{(E)-Butyl 3-(2,5-dibromophenyl)acrylate (27)}

From $n$-butylacrylate $(0.192 \mathrm{~g}, 1.5 \mathrm{mmol})$ and 1,4-dibromobenzene-1-sulfonyl chloride (0.334 g, $1 \mathrm{mmol})$, product 27 was obtained in $91 \%(0.329 \mathrm{~g})$ yield as a colourless oil.

$\left.{ }^{1} \mathrm{H} \mathrm{NMR} \mathrm{(400} \mathrm{MHz,} \mathrm{CDCl}_{3}\right) \delta 7.93(\mathrm{~d}, J=16.0 \mathrm{~Hz}, 1 \mathrm{H}), 7.72(\mathrm{~d}, J=2.4 \mathrm{~Hz}, 1 \mathrm{H}), 7.46(\mathrm{~d}, J=8.3 \mathrm{~Hz}, 1 \mathrm{H}), 7.29(\mathrm{dd}, J=8.3,2.4 \mathrm{~Hz}, 1 \mathrm{H}), 6.39(\mathrm{~d}, J$ $=16.0 \mathrm{~Hz}, 1 \mathrm{H}$ ), $4.24(\mathrm{t}, J=7.6 \mathrm{~Hz}, 2 \mathrm{H}), 1.68$ (quint., $J=7.6 \mathrm{~Hz}, 2 \mathrm{H}$ ), 1.41 (sext., $J=7.6 \mathrm{~Hz}, 2 \mathrm{H}$ ), $0.97(\mathrm{t}, J=7.6 \mathrm{~Hz}, 3 \mathrm{H}$ ).

${ }^{13} \mathrm{C}$ NMR $\left(100 \mathrm{MHz}, \mathrm{CDCl}_{3}\right) \delta 166.9,141.5,136.5,134.7,133.9,130.6,123.8,122.4,121.7,64.8,30.8,19.3,13.8$.

$\mathrm{C}_{13} \mathrm{H}_{14} \mathrm{Br}_{2} \mathrm{O}_{2}$ (362.06): Calcd C 43.13, H 3.90; Found C 43.01, H 3.87.

\section{(E)-Butyl 3-(3,4-dibromophenyl)acrylate (28)}

From $n$-butylacrylate $(0.192 \mathrm{~g}, 1.5 \mathrm{mmol})$ and 3,4-dibromobenzene-1-sulfonyl chloride $(0.334 \mathrm{~g}, 1 \mathrm{mmol})$, product 28 was obtained in $59 \%(0.214 \mathrm{~g})$ yield as a yellow solid (mp: $\left.38-41^{\circ} \mathrm{C}\right)$.

${ }^{1} \mathrm{H} \mathrm{NMR}\left(400 \mathrm{MHz}, \mathrm{CDCl}_{3}\right) \delta 7.76(\mathrm{~d}, J=2.1 \mathrm{~Hz}, 1 \mathrm{H}), 7.62(\mathrm{~d}, J=8.3 \mathrm{~Hz}, 1 \mathrm{H}), 7.54(\mathrm{~d}, J=16.0 \mathrm{~Hz}, 1 \mathrm{H}), 7.30(\mathrm{dd}, J=8.3,2.1 \mathrm{~Hz}, 1 \mathrm{H}), 6.43(\mathrm{~d}, J$ $=16.0 \mathrm{~Hz}, 1 \mathrm{H}$ ), $4.21(\mathrm{t}, J=7.6 \mathrm{~Hz}, 2 \mathrm{H}$ ), 1.68 (quint., $J=7.6 \mathrm{~Hz}, 2 \mathrm{H}$ ), 1.41 (sext., $J=7.6 \mathrm{~Hz}, 2 \mathrm{H}), 0.95(\mathrm{t}, J=7.6 \mathrm{~Hz}, 3 \mathrm{H}$ ).

${ }^{13} \mathrm{C}$ NMR $\left(100 \mathrm{MHz}, \mathrm{CDCl}_{3}\right) \delta 166.8,141.8,135.4,134.2,132.9,127.7,126.6,125.6,120.4,64.8,30.9,19.3,13.9$.

$\mathrm{C}_{13} \mathrm{H}_{14} \mathrm{Br}_{2} \mathrm{O}_{2}$ (362.06): Calcd C 43.13, H 3.90; Found C 43.20, H 3.99.

\section{(E)-n-Butyl 3-(4-iodophenyl)acrylate (29) ${ }^{7}$}

From $n$-butylacrylate $(0.192 \mathrm{~g}, 1.5 \mathrm{mmol})$ and 4-iodobenzenesulfonyl chloride $(0.303 \mathrm{~g}, 1 \mathrm{mmol})$, product 29 was obtained in $82 \%$ $(0.271 \mathrm{~g})$ yield as a white solid (mp: $\left.39-41^{\circ} \mathrm{C}\right)$.

$\left.{ }^{1} \mathrm{H} \mathrm{NMR} \mathrm{(400} \mathrm{MHz,} \mathrm{CDCl} 3\right) \delta 7.70(\mathrm{~d}, J=8.3 \mathrm{~Hz}, 2 \mathrm{H}), 7.57(\mathrm{~d}, J=16.0 \mathrm{~Hz}, 1 \mathrm{H}), 7.23(\mathrm{~d}, J=8.3 \mathrm{~Hz}, 2 \mathrm{H}), 6.43(\mathrm{~d}, J=16.0 \mathrm{~Hz}, 1 \mathrm{H}), 4.20(\mathrm{t}, J=$ $7.6 \mathrm{~Hz}, 2 \mathrm{H}$ ), 1.68 (quint., $J=7.6 \mathrm{~Hz}, 2 \mathrm{H}$ ), 1.41 (sext., $J=7.6 \mathrm{~Hz}, 2 \mathrm{H}$ ), 0.95 (t, $J=7.6 \mathrm{~Hz}, 3 \mathrm{H}$ ).

${ }^{13} \mathrm{C}$ NMR $\left(100 \mathrm{MHz}, \mathrm{CDCl}_{3}\right) \delta 166.8,143.4,138.2,134.0,129.6,119.2,96.5,64.6,30.8,19.3,13.8$.

\section{(E)-Butyl 3-(2-iodo-5-nitrophenyl)acrylate (30)}

From $n$-butylacrylate $(0.192 \mathrm{~g}, 1.5 \mathrm{mmol})$ and 2-iodo-5-nitrobenzene-1-sulfonyl chloride ( $0.347 \mathrm{~g}, 1 \mathrm{mmol})$, product $\mathbf{3 0}$ was obtained in $28 \%(0.105 \mathrm{~g})$ yield as a yellow oil.

${ }^{1} \mathrm{H}$ NMR $\left(400 \mathrm{MHz}, \mathrm{CDCl}_{3}\right) \delta 8.30(\mathrm{~d}, J=2.3 \mathrm{~Hz}, 1 \mathrm{H}), 8.14(\mathrm{dd}, J=8.7,2.3 \mathrm{~Hz}, 1 \mathrm{H}), 8.04(\mathrm{~d}, J=16.0 \mathrm{~Hz}, 1 \mathrm{H}), 7.77(\mathrm{~d}, J=8.7 \mathrm{~Hz}, 1 \mathrm{H}), 6.55(\mathrm{~d}, J$ $=16.0 \mathrm{~Hz}, 1 \mathrm{H})$.

${ }^{13} \mathrm{C}$ NMR $\left(100 \mathrm{MHz}, \mathrm{CDCl}_{3}\right) \delta 165.8,148.6,139.2,138.2,135.6,128.4,125.5,125.0,122.1,65.2,30.8,19.3,13.9$.

$\mathrm{C}_{13} \mathrm{H}_{14} \mathrm{NIO}_{4}$ (375.16): Calcd C 41.62, H 3.76; Found C 41.66, H 3.99.

\section{(E)-Ethyl 3-(4-bromophenyl)but-2-enoate (31) ${ }^{29}$}

From ethyl trans-2-butenoate $(0.171 \mathrm{~g}, 1.5 \mathrm{mmol})$ and 4-bromobenzenesulfonyl chloride $(0.255 \mathrm{~g}, 1 \mathrm{mmol})$, product 31 was obtained in $20 \%(0.054 \mathrm{~g})$ yield as a colourless oil.

${ }^{1} \mathrm{H}$ NMR (400 MHz, $\left.\mathrm{CDCl}_{3}\right) \delta 7.50(\mathrm{~d}, J=6.5 \mathrm{~Hz}, 2 \mathrm{H}), 7.34(\mathrm{~d}, J=6.5 \mathrm{~Hz}, 2 \mathrm{H}), 6.11(\mathrm{~s}, 1 \mathrm{H}), 4.21(\mathrm{q}, J=6.7 \mathrm{~Hz}, 2 \mathrm{H}), 2.54(\mathrm{~d}, J=1.3 \mathrm{~Hz}, 3 \mathrm{H})$, $1.31(\mathrm{t}, J=6.7 \mathrm{~Hz}, 3 \mathrm{H})$.

${ }^{13} \mathrm{C}$ NMR $\left(100 \mathrm{MHz}, \mathrm{CDCl}_{3}\right) \delta 166.8,154.2,141.2,131.8,128.0,123.3,117.8,60.1,17.9,14.5$.

\section{(E)-1-Bromo-4-(3,3-dimethylbut-1-enyl)benzene (32) ${ }^{30}$}

From 3,3-dimethylbut-1-ene ( $0.420 \mathrm{~g}, 5 \mathrm{mmol}$ ) and 4-bromobenzenesulfonyl chloride $(0.255 \mathrm{~g}, 1 \mathrm{mmol})$, product 32 was obtained in $36 \%(0.086 \mathrm{~g})$ yield as a white solid (mp: $\left.62-65^{\circ} \mathrm{C}\right)$.

$\left.{ }^{1} \mathrm{H} \mathrm{NMR} \mathrm{(400} \mathrm{MHz,} \mathrm{CDCl}_{3}\right) \delta 7.40(\mathrm{~d}, J=6.3 \mathrm{~Hz}, 2 \mathrm{H}), 7.22(\mathrm{~d}, J=6.3 \mathrm{~Hz}, 2 \mathrm{H}), 6.24(\mathrm{~s}, 2 \mathrm{H}), 1.12(\mathrm{~s}, 9 \mathrm{H})$.

${ }^{13} \mathrm{C}$ NMR $\left(100 \mathrm{MHz}, \mathrm{CDCl}_{3}\right) \delta 142.8,137.2,131.6,127.7,123.7,120.5,33.6,29.6$.

\section{(E)-(4-Iodostyryl)dimethyl(phenyl)silane (33)}

From dimethyl(phenyl)(vinyl)silane $(0.243 \mathrm{~g}, 1.5 \mathrm{mmol})$ and 4-iodobenzenesulfonyl chloride (0.303 g, 1 mmol), product 33 was obtained in $31 \%$ (0.113 g) yield as a colourless oil. 
${ }^{1} \mathrm{H}$ NMR $\left(400 \mathrm{MHz}, \mathrm{CDCl}_{3}\right) \delta 7.66(\mathrm{~d}, J=8.3 \mathrm{~Hz}, 2 \mathrm{H}), 7.60-7.53(\mathrm{~m}, 2 \mathrm{H}), 7.40-7.35(\mathrm{~m}, 3 \mathrm{H}), 7.18(\mathrm{~d}, J=8.3 \mathrm{~Hz}, 2 \mathrm{H}), 6.85(\mathrm{~d}, J=19.1 \mathrm{~Hz}, 1 \mathrm{H})$, $6.59(\mathrm{~d}, J=19.1 \mathrm{~Hz}, 1 \mathrm{H}), 0.44(\mathrm{~s}, 6 \mathrm{H})$.

${ }^{13} \mathrm{C}$ NMR $\left(100 \mathrm{MHz}, \mathrm{CDCl}_{3}\right) \delta 144.2,138.3,137.8,137.7,134.0,129.3,128.6,128.4,128.0,93.8,-2.5$.

$\mathrm{C}_{16} \mathrm{H}_{17} \mathrm{ISi}$ (364.30): Calcd C 52.75, H 4.70; Found C 52.64, H 4.48.

\section{(E)-2-Ethyl-4-methyl-5-(4-styrylphenyl)thiazole (34)}

(E)-1-Bromo-4-styrylbenzene 1 (0.259 g, $1 \mathrm{mmol}$ ), 2-ethyl-4-methylthiazole (0.191 g, $1.5 \mathrm{mmol})$, KOAc (0.196 g, $2 \mathrm{mmol})$, and $\mathrm{PdCl}\left(\mathrm{C}_{3} \mathrm{H}_{5}\right)(\mathrm{dppb})(12.2 \mathrm{mg}, 0.02 \mathrm{mmol})$, were dissolved in DMA $(2 \mathrm{~mL})$ under an argon atmosphere. The reaction mixture was stirred at $130{ }^{\circ} \mathrm{C}$ for $24 \mathrm{~h}$. After evaporation of the solvent, the product was purified by silica gel column chromatography affording $\mathbf{3 4}$ in $53 \%$ $(0.162 \mathrm{~g})$ yield as a yellow solid (mp: $\left.112-114^{\circ} \mathrm{C}\right)$.

${ }^{1} \mathrm{H}$ NMR $\left(400 \mathrm{MHz}, \mathrm{CDCl}_{3}\right) \delta 7.58(\mathrm{~d}, J=8.5 \mathrm{~Hz}, 2 \mathrm{H}), 7.56(\mathrm{~d}, J=8.5 \mathrm{~Hz}, 2 \mathrm{H}), 7.47-7.35(\mathrm{~m}, 4 \mathrm{H}), 7.30(\mathrm{t}, J=7.4 \mathrm{~Hz}, 1 \mathrm{H}), 7.19(\mathrm{~d}, J=16.4 \mathrm{~Hz}$, $1 \mathrm{H}), 7.14(\mathrm{~d}, J=16.4 \mathrm{~Hz}, 1 \mathrm{H}), 3.04(\mathrm{q}, J=7.6 \mathrm{~Hz}, 2 \mathrm{H}), 2.53(\mathrm{~s}, 3 \mathrm{H}), 1.44(\mathrm{t}, J=7.6 \mathrm{~Hz}, 3 \mathrm{H})$.

${ }^{13} \mathrm{C}$ NMR $\left(100 \mathrm{MHz}, \mathrm{CDCl}_{3}\right) \delta 170.4,147.1,137.3,136.8,131.7,130.9,129.5,129.4,128.9,128.0,127.9,126.8,126.7,27.1,16.4,14.5$.

$\mathrm{C}_{20} \mathrm{H}_{19} \mathrm{NS}$ (305.44): Calcd C 78.65, H 6.27; Found C 78.79, H 6.09.

\section{(E)-2-Pentyl-5-(4-styrylphenyl)thiophene (35)}

(E)-1-Bromo-4-styrylbenzene 1 (0.259 g, 1 mmol), 2-pentylthiophene (0.231 g, $1.5 \mathrm{mmol})$, KOAc (0.196 g, 2 mmol), and $\mathrm{PdCl}\left(\mathrm{C}_{3} \mathrm{H}_{5}\right)(\mathrm{dppb})(12.2 \mathrm{mg}, 0.02 \mathrm{mmol})$, were dissolved in DMA $(2 \mathrm{~mL})$ under an argon atmosphere. The reaction mixture was stirred at $130{ }^{\circ} \mathrm{C}$ for $24 \mathrm{~h}$. After evaporation of the solvent, the product was purified by silica gel column chromatography affording $\mathbf{3 5}$ in $51 \%$ $(0.169 \mathrm{~g})$ yield as a brown solid (mp: $\left.190-192^{\circ} \mathrm{C}\right)$.

${ }^{1} \mathrm{H} \mathrm{NMR}\left(400 \mathrm{MHz}, \mathrm{CDCl}_{3}\right) \delta 7.60-7.46(\mathrm{~m}, 6 \mathrm{H}), 7.37(\mathrm{t}, J=7.4 \mathrm{~Hz}, 2 \mathrm{H}), 7.26(\mathrm{t}, J=7.4 \mathrm{~Hz}, 1 \mathrm{H}), 7.15(\mathrm{~d}, J=3.5 \mathrm{~Hz}, 1 \mathrm{H}), 7.11(\mathrm{~s}, 2 \mathrm{H}), 6.75(\mathrm{~d}, J$ $=3.5 \mathrm{~Hz}, 1 \mathrm{H}), 2.81(\mathrm{t}, J=7.6 \mathrm{~Hz}, 2 \mathrm{H}), 1.77-1.65(\mathrm{~m}, 2 \mathrm{H}), 1.44-1.33(\mathrm{~m}, 4 \mathrm{H}), 0.92(\mathrm{t}, J=7.6 \mathrm{~Hz}, 3 \mathrm{H})$.

${ }^{13} \mathrm{C}$ NMR $\left(100 \mathrm{MHz}, \mathrm{CDCl}_{3}\right) \delta 146.0,141.5,137.5,136.1,134.1,128.8,128.5,128.3,127.7,127.1,126.6,125.7,125.2,122.8,31.5,31.4$, $30.4,22.6,14.2$.

$\mathrm{C}_{23} \mathrm{H}_{24} \mathrm{~S}$ (332.50): Calcd C 83.08, H 7.28; Found C 82.94, H 7.30.

\section{Acknowledgment}

We thank the Centre National de la Recherche Scientifique, Rennes Metropole and Scientific Ministry of Higher Education and Research of Tunisia for providing financial support.

\section{Supporting Information}

YES (this text will be updated with links prior to publication)

\section{References}

(1) (a) de Vries J. G. Can. J. Chem. 2001, 79, 1086-1092; (b) Oestreich, M. The Mizoroki-Heck Reaction, Wiley, New York, 2009; (c) Le Bras J.; Muzart, J. Chem. Rev., 2011, 111, 1170-1214; (d) Mc Cartney, D.; Guiry, P. J. Chem; Soc. Rev. 2011, 40, 5122-5150; (e) Hussain, M.; Van Sung, T.; Langer, P. Synlett 2012, 23, 2735-2745.

(2) For Heck type reactions with aryl sulfinic acids: Wang G.-W.; Miao, T. Chem. Eur. J., 2011, 17, 5787-5790.

(3) For Heck type reaction with benzenesulfonyl chlorides: (a) Miura, M.; Hashimoto, H.; Itoh K.; Nomura, M. Tetrahedron Lett., 1989, 30, 975-976; (b) Dubbaka, S. R.; Zhao, D.; Fei, Z.; Volla, C. M. R.; Dyson P. J.; Vogel, P. Synlett, 2006, 3155-3157; (c) Jafarpour, F.; Olia M. B. A.; Hazrati, H. Adv. Synth. Catal., 2013, 355, 3407-3412; (d) Kusunuru, A. K.; Yousuf, S. K.; Tatina, M.; Mukherjee, D. Eur. J. Org. Chem. 2015, 459-462.

(4) For a review on metal-catalysed desulfitative direct arylation: Yuan, K.; Soulé J. F.; Doucet, H. ACS Catal., $2015,5,978-991$.

(5) For a single example of Pd-catalysed Heck type reaction with a 4-bromobenzenesulfonyl chloride: (a) Miura, M.; Hashimoto, H.; Itoh K.; Nomura, M. J. Chem. Soc., Perkin Trans. 1, 1990, 2207-2211; For an example of Pd-catalysed Heck type reaction with a 4-bromobenzenesulfinate : (b) Zhou, X.; Luo, J.; Liu, J.; Peng S.; Deng, G.-J. Org. Lett., 2011, 13, 1432-1435; (c) Hu, S.; Xia, P.; Cheng K.; Qi, C. Appl. Organomet. Chem., 2013, 27, 188-190. For examples of Pd-catalysed Heck type reaction with a 4-bromobenzenesulfonyl hydrazide: (d) Yang, F.-L.; Ma X.-T.; Tian, S.-K. Chem. Eur. J., 2012, 18, 1582-1585.

(6) For a single example of Rh-catalysed Heck type reaction with a 4-bromobenzenesulfonyl chloride: Dubbaka S. R.; Vogel, P. Chem. Eur. J., 2005, 11, 2633-2641.

(7) For examples of Ru-catalysed Heck type reaction with halo-substituted arylboronic acids: Farrington, E. J.; Barnard, C. F. J.; Rowsell, E.; Brown, J. M. Adv. Synth. Catal. 2005, 347, 185-195.

(8) For a review on transition-metal mediated C-S bond activation: Wang, L.; He W.; Yu, Z. Chem. Soc. Rev., 2013, 42, 599-921.

(9) For selected examples of Pd-catalysed direct arylations via desulfitative coupling from our laboratory: (a) Yuan K.; Doucet, H. Chem. Sci., 2014, 5, 392-396; (b) Loukotova, L.; Yuan, K.; Doucet, H. ChemCatChem, 2014, 6, 1303-1309; (c) Hfaiedh, A.; Yuan, K.; Ben Ammar, H.; Ben Hassine, B.; Soulé, J.-F.; Doucet H. ChemSusChem 2015, 8, 1794-1804.

(10) For Pd-catalysed conjugate addition using benzenesulfonyl chlorides and enones: Yuan, K.; Sang, R.; Soulé, J.-F.; Doucet H. Cat. Sci. Technol. 2015, 5, 2904-2912.

(11) Amatore, C.; Jutand A.; Le Duc, G. Chem. Eur. J. 2012, 18, 6616-6625. 
(12) (a) Loeser, R.; Bergmann, R.; Frizler, M.; Mosch, B.; Dombrowski, L.; Kuchar, M.; Steinbach, J.; Guetschow, M.; Pietzsch, J. ChemMedChem 2013, 8, 1330-1344; (b) Nielsen, C. B.; Johnsen, M.; Arnbjerg, J.; Pittelkow, M.; Mcllroy, S. P.; Ogilby, P. R.; Jorgensen, M. J. Org. Chem. 2005, 70, 7065-7079; (c) Biet, T.; Fihey, A.; Cauchy, T.; Vanthuyne, N.; Roussel, C.; Crassous, J.; Avarvari, N. Chem. Eur. J. 2013, 19, 13160-13167.

(13) For selected examples of Heck type reactions with disubstituted alkenes: (a) Melpolder, J.; Heck, R. J. Org. Chem. 1976, 41, 265-272; (b) Cortese N.; Ziegler, C.; Hrnjez, B., Heck, R. J. Org. Chem. 1978, 43, 2952-2958; (c) Cacchi, S.; Arcadi, A. J. Org. Chem. 1983, 48, 4236-4270; (d) Amorese, A.; Arcadi, A.; Bernocchi, E.; Cacchi, S.; Cerrini, S.; Fereli, W.; Ortar, G. Tetrahedron 1989, 45, 813-828; (e) Beller, M.; Riermeier, T. Tetrahedron Lett. 1996, 37, 6535-6538; (f) Beller, M.; Riermeier, T. Eur. J. Inorg. Chem. 1998, 1, 29-35; (g) Netherton, M.; Fu, G. Org. Lett. 2001, 3, 4295-4298; (h) Berthiol, F; Doucet, H; Santelli, M Eur. J. Org. Chem. 2003, 1091-1096; (i) Kondolff, I, Doucet, H; Santelli, M. Tetrahedron Lett. 2003, 44, 8487-8491.

(14) Zhao X.; Dong, V. M. Angew. Chem., Int. Ed. 2011, 50, 932-934.

(15) For reviews: (a) Satoh, T.; Miura, M. Chem. Lett. 2007, 36, 200-205; (b) Li, B.-J.; Yang, S.-D.; Shi, Z.-J. Synlett 2008, 949-957; (c) Bellina, F.; Rossi, R. Tetrahedron 2009, 65, 10269-10310; (d) Ackermann, L.; Vicente, R.; Kapdi, A. Angew. Chem. Int. Ed. 2009, 48, 9792-9826; (e) Joucla, L.; Djakovitch, L. Adv. Synth. Catal. 2009, 351, 673-714; (f) Ackermann, L. Chem. Rev. 2011, 111, 1315-1345; (g) Kuhl, N.; Hopkinson, M. N.; Wencel-Delord, J.; Glorius, F. Angew. Chem. Int. Ed. 2012, 51, 10236-10254; (h) Neufeldt, S. R.; Sanford, M. S. Acc. Chem. Res. 2012, 45, 936-946; (i) Wencel-Delord, J.; Glorius, F. Nature Chem. 2013, 5, 369-375; (j) Rossi, R.; Bellina, F.; Lessi, M.; Manzini, C. Adv. Synth. Catal. 2014, 356, 17-117; (k) He, M.; Soulé, J.-F.; Doucet, H. ChemCatChem 2014, 6, 1824-1859; (l) Miura, M.; Satoh, T.; Hirano, K. Bull. Chem. Soc. Jpn 2014, 87, 751-764; (m) Bheeter, C. B.; Chen, L.; Soulé, J.-F.; Doucet, H. Cat. Sci. Technol. 2016, DOI:10.1039/C5CY02095F.

(16) Cantat, T.; Génin, E.; Giroud, C.; Meyer G.; Jutand, A. J. Organomet. Chem. 2003, 687, 365-376.

(17) Sugihara, T.; Satoh, T.; Miura, M.; Nomura, M. Adv. Synth. Catal. 2004, 346, 1765-1772.

(18) Cella, R.; Stefani, H. A. Tetrahedron 2006, 62, 5656-5662.

(19) Berthiol, F.; Kondolff, I.; Doucet, H.; Santelli, M. J. Org. Chem. 2004, 689, 2786-2798.

(20) Kohno, T.; Togashi, K.; Fukamiya, N. Nat. Prod. Res. 2007, 21, 606-615.

(21) Gigante, B.; Esteves, M. A.; Pires, N.; Davies, M. L.; Douglas, P.; Fonseca, S. M.; Burrows, H. D.; Castro, R. A. E.; Pina, J.; Seixas de Melo, J. New J. Chem. 2009, 33, 877-885.

(22) Yang, J.-S.; Hwang, C.-Y.; Hsieh, C.-C.; Chiou, S.-Y. J. Org. Chem. 2004, 69, 719-726.

(23) Patureau, F. W.; Nimphius, C.; Glorius, F. Org. Lett. 2011, 13, 6346-6349.

(24) Aydin, J.; Larsson, J. M.; Selander, N.; Szabo, K. J. Org. Lett. 2009, 11, 2852-2854.

(25) Kalkhambkar, R. G.; Laali, K. K. Tetrahedron Lett. 2011, 52, 1733-1737.

(26) Aun, C. E.; Clarkson, T. J.; Happer, D. A. R. J. Chem. Soc., Perkin Trans. 2 1990, 645-649.

(27) Wang, H.-W.; Yeh, M.-Y.; Chen, C.-H.; Lim, T.-S.; Fann, W.; Luh, T.-Y. Macromolecules 2008, 41, 2762-2770.

(28) Ebraheem, K. A. K.; Webb, G. A. J. Mol. Struct. 1975, 25, 387-96.

(29) Metternich, J. B.; Gilmour R. J. Am. Chem. Soc. 2015, 137, 11254-11257.

(30) Mai, W.-P.; Song, G.; Sun, G.-C.; Yang, L.-R.; Yuan, J.-W.; Xiao, Y.-M.; Mao, P.; Qu, L.-B. RSC Advances 2013, 3, 19264-19267. 